\title{
Matrices of Formal Power Series Associated to Binomial Posets
}

\author{
GÁBOR HETYEI* \\ Mathematics Department, UNC Charlotte, Charlotte, NC 28223
}

Received June 17, 2003; Revised June 17, 2003; Accepted November 11, 2004

\begin{abstract}
We introduce an operation that assigns to each binomial poset a partially ordered set for which the number of saturated chains in any interval is a function of two parameters. We develop a corresponding theory of generating functions involving noncommutative formal power series modulo the closure of a principal ideal, which may be faithfully represented by the limit of an infinite sequence of lower triangular matrix representations. The framework allows us to construct matrices of formal power series whose inverse may be easily calculated using the relation between the Möbius and zeta functions, and to find a unified model for the Tchebyshev polynomials of the first kind and for the derivative polynomials used to express the derivatives of the secant function as a polynomial of the tangent function.
\end{abstract}

Keywords: partially ordered set, binomial, noncommutative formal power series, Tchebyshev polynomial, derivative polynomial

\section{Introduction}

In a recent paper [6] the present author introduced a sequence of Eulerian partially ordered sets whose $c e$-indices provide a noncommutative generalization of the Tchebyshev polynomials. The partially ordered sets were obtained by looking at intervals in a poset obtained from the simplest possible infinite lower Eulerian poset represented in figure 1 and using an operator that could be applied to any partially ordered set. This operator, which we call the Tchebyshev operator, creates a partial order on the non-singleton intervals of its input, by setting $\left(x_{1}, y_{1}\right) \leq\left(x_{2}, y_{2}\right)$ when either $y_{1} \leq x_{2}$, or $x_{1}=x_{2}$ and $y_{1} \leq y_{2}$. The property of having a rank function is preserved by the Tchebyshev operator. The existence of a unique minimum element is not preserved, but if we "augment" the poset that has a unique minimum element $\hat{0}$ by adding a new minimum element $\widehat{-1}$, then the Tchebyshev transform of the augmented poset will have a unique minimum element associated to the interval $(\widehat{-1}, \hat{0})$.

In this paper we study the effect of the augmented Tchebyshev operator on binomial posets. As it is well known, binomial posets provide a framework for studying generating functions. Those functions of the incidence algebra that depend only on the rank of the interval, form a subalgebra, and there is a homomorphism from this subalgebra into the ring of formal power series in one variable. Combinatorial enumeration problems stated in terms 


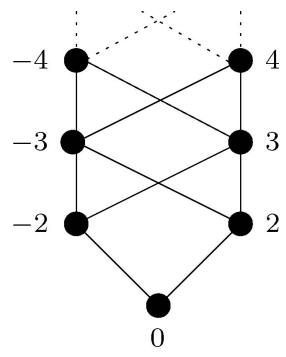

Figure 1. The "ladder" poset.

of binomial posets may be solved using generating functions and, conversely, identities of formal power series may be explained by exposing the combinatorial background.

The augmented Tchebyshev transform of a binomial poset is never binomial (this is shown in Section 4) but, as far as the enumeration of saturated chains is concerned, each interval may be characterized by a pair of integers. This description, together with the generalizations of the factorial functions and of the binomial coefficients, are presented in Sections 2 and 3. Hence it is a natural generalization of the theory of binomial posets to consider those functions in the incidence algebra of their augmented Tchebyshev transform which are constant on intervals of the same type. In Section 4 we define a ring of generating functions (called the Tchebyshev algebra) that is isomorphic to the subalgebra of these functions. In Section 5 we show that our ring of generating functions is isomorphic to the ring of noncommutative formal power series in $x$ and $y$, modulo the closure of the ideal generated by $y x-x^{2}$. The resulting ring is more complex than the ring of formal power series in one variable, and there are infinitely many ways to represent it as a ring of $d \times d$ matrices whose entries are formal power series in one variable. We construct a series of $d \times d$ matrix representations, each representation being a lift of the previous one, such that the "limit representation" of infinite lower triangular matrices is a faithful representation.

Using our matrix representations, any relation that holds for functions that are constant on intervals of the same type may be translated into a relation between matrices of formal power series. In Sections 6 and 7 we describe a few translations of the fact that the zeta function is the multiplicative inverse of the Möbius function, and obtain formulas for inverting some nontrivial matrices of formal power series. Since the augmented Tchebyshev transform of a lower Eulerian poset is lower Eulerian, in the case of lower Eulerian binomial posets we obtain a particularly elegant rule: to invert the matrix associated to the zeta function, one needs to substitute $(-t)$ into the variable $t$ in each entry of the matrix.

Describing all matrix representations of the Tchebyshev algebra is beyond the scope of this paper. In Section 8 we describe at least all "one-dimensional representations", that is all homomorphisms from the Tchebyshev algebra into a ring of formal power series $K[[t]]$ in one variable. It turns out that, modulo the endomorphisms of $K[[t]]$, there are only two essentially different homomorphisms. One of them may be extended to a homomorphism from the incidence algebra of the augmented Tchebyshev transform of an arbitrary poset into the incidence algebra of the same poset, the other seems to extend only to the level of standard algebras. 
Finally, in Section 9 we show that the augmented Tchebyshev transform may be used to generalize the notion of Tchebyshev polynomials and establish links to combinatorially interesting polynomial sequences. The same operation that associates to the "ladder" poset in figure 1 the Tchebyshev polynomials of the first kind, associates to the poset of finite subsets of an infinite set the derivative polynomials used to express the derivatives of the secant function as a polynomial of the tangent function. These polynomials may be used to express several combinatorially important integer sequences, as it is described in Hoffman's paper [9].

The results of this paper mark only the tip of an iceberg which is yet to be explored. There are many more results on functions that depend on the rank of an interval only in the incidence algebra of a binomial poset, than just the relation between the Möbius function and the zeta function. Some examples of such relations are given in section 3.15 of Stanley's book [15]. Analogous formulas for the augmented Tchebyshev transform will yield formulas for matrices of formal power series. Moreover, since we had a lot of freedom in choosing the matrices representing our variables $x$ and $y$, other matrix representations of the Tchebyshev algebra are yet to be discovered, which may yield even more results.

Finally, one may want to ask whether there are other operators, analogous to the Tchebyshev operator, which would yield similar results in rings derived from the ring of formal power series.

\section{Preliminaries}

\subsection{Binomial posets}

A partially ordered set $P$ is locally finite if every interval $[x, y] \subseteq P$ contains a finite number of elements. An element $y \in P$ covers $x \in P$ if $y>x$ and there is no element between $x$ and $y$. We will use the notation $y \succ x$. A function $\rho: P \rightarrow \mathbb{Z}$ is a rank function for $P$ if $\rho(y)=\rho(x)+1$ is satisfied whenever $y$ covers $x$. A partially ordered set may have more than one rank function, but the restriction of any rank function to any interval $[x, y] \subseteq P$ is unique up to a constant shift. Therefore the rank $\rho(x, y)$ of an interval $[x, y]$, defined by $\rho(x, y)=\rho(y)-\rho(x)$, is the same number for any rank function, and it is equal to the common length of all maximal chains connecting $x$ and $y$. We say that a finite partially ordered set is graded when it has a unique minimum element, a unique maximum element, and a rank function $\rho$. A locally finite partially ordered set $P$ is binomial, if it has a unique minimum element $\hat{0}$, contains an infinite chain, every interval $[x, y] \subseteq P$ is graded, and the number $B(n)$ of saturated chains from $x$ to $y$ depends only on $n=\rho(x, y)$. The function $B(n)$ is called the factorial function of $P$.

Binomial posets are a natural tool to generalize the notion of exponential generating functions. Given any locally finite poset $P$, the incidence algebra $I(P, K)$ of $P$ over a field $K$ consists of all functions $f: \operatorname{Int}(P) \rightarrow K$ mapping the set of intervals of $P$ into $K$, together with pointwise addition and the multiplication rule

$$
(f \cdot g)([x, y])=\sum_{x \leq z \leq y} f([x, z]) g([z, y]) .
$$


This multiplication is often called convolution. Those functions of the incidence algebra which depend only on the rank of the interval form a subalgebra $R(P, K)$. For its elements $f \in R(P, K)$ we may write (by abuse of notation) $f(n)$ instead of $f([x, y])$ where $[x, y] \subseteq$ $P$ is any interval of rank $n$. Then we have the following multiplication rule

$$
(f \cdot g)(n)=\sum_{k=0}^{n}\left[\begin{array}{l}
n \\
k
\end{array}\right] f(k) g(n-k),
$$

where $\left[\begin{array}{l}n \\ k\end{array}\right]$ is the "binomial coefficient" $\frac{B(n)}{B(k) B(n-k)}$. As a consequence of this formula, it is easy to show that associating to each $f \in R(P, K)$ the formal power series

$$
\phi(f)=\sum_{n=0}^{\infty} \frac{f(n)}{B(n)} x^{n} \in K[[x]]
$$

yields an algebra homomorphism from $R(P, K)$ into $K[[x]]$. The details of this theory are well explained in Stanley's book [15, Section 3.15]. Generalizations were developed by Ehrenborg and Readdy in [3], and by Reiner in [13].

\subsection{Möbius function and Eulerian posets}

The zeta function $\zeta \in I(P, K)$ is the function whose value is 1 on every interval of $P$. The Möbius function $\mu$ is the multiplicative inverse of the zeta function. In other words, the value of the Möbius function may be recursively defined, by $\mu([x, x])=1$ and $\sum_{x \leq z \leq y} \mu([x, z])=0$ for all intervals $[x, y]$ satisfying $x<y$. A graded partially ordered set is Eulerian if every interval $[x, y]$ in it satisfies $\mu([x, y])=(-1)^{\rho(x, y)}$.

Following [17] we call a partially ordered set $P$ lower Eulerian if it has a unique minimum element $\hat{0}$ and for every $u \in P$ the interval $[\hat{0}, u]$ is Eulerian.

If a partially ordered set is binomial, then the zeta function and the Möbius function both belong to $R(P, K)$. If the partially ordered set is also lower Eulerian, then the homomorphism $\phi: R(P, K) \rightarrow K[[x]]$ introduced in Section 1.1 provides evidence that the formal power series $\sum_{n \geq 0} x^{n} / B(n)$ and $\sum_{n \geq 0}(-1)^{n} x^{n} / B(n)$ are multiplicative inverses of each other.

\subsection{General Tchebyshev posets}

In [6] we define the (general) Tchebyshev poset $T(Q)$ associated to an arbitrary locally finite poset $Q$ as follows. Its elements are all ordered pairs $(x, y) \in Q \times Q$ satisfying $x<y$, and we set $\left(x_{1}, y_{1}\right) \leq\left(x_{2}, y_{2}\right)$ when $y_{1} \leq x_{2}$, or $x_{1}=x_{2}$ and $y_{1} \leq y_{2}$.

It is natural to think of the elements of $T(Q)$ as the non-singleton intervals $[x, y]$ of $Q$. We consider an interval larger than the other if either every element of the larger interval is larger than every element of the smaller interval or the smaller interval is an "initial segment" of the larger interval. 
Although most of the paper [6] focuses on the intervals of $T(Q)$ for a specific $Q$, the following statements were shown for arbitrary locally finite posets (see Propositions 2.2, 2.3, 1.4, Lemma 1.5, and Proposition 1.6 in [6]):

Proposition 1.1 $T(Q)$ is a partially ordered set.

Proposition 1.2 If $\rho: Q \rightarrow \mathbb{Z}$ is a rank function for $Q$ then setting $\rho(x, y)=\rho(y)$ provides a rank function for $T(Q)$. In fact, the set of elements covering $(x, y) \in T(Q)$ is

$$
\{(x, \dot{y}): y \prec \dot{y}\} \cup\{(y, \dot{y}): y \prec \dot{y}\} \text {. }
$$

(Here $\dot{y}$ denotes an arbitrary element covering y in $Q$.)

Proposition 1.3 Assume that every element of $Q$ is comparable to at least one other element of $Q$. Then $T(Q)$ has a unique minimum element if and only if $Q$ has a unique minimum element $x_{0}$ covered by a unique atom $y_{0}$. In that case the unique minimum element of $T(Q)$ is $\left(x_{0}, y_{0}\right)$.

Lemma 1.4 Given $x_{1}<y_{1} \leq y_{2} \in Q$, the interval $\left[\left(x_{1}, y_{1}\right),\left(x_{1}, y_{2}\right)\right] \subseteq T(Q)$ is isomorphic to $\left[y_{1}, y_{2}\right] \subseteq Q$.

Proposition 1.5 Assume that every element of $Q$ is comparable to some other element and that $T(Q)$ has a unique minimum element $\left(x_{0}, y_{0}\right)$. Then every interval of $T(Q)$ is an Eulerian poset if and only the same holds for every interval of $Q \backslash\left\{x_{0}\right\}$.

Proposition 1.3 suggests considering the following modified version of the operation $T$ when it is applied to a poset that has a unique minimum element.

Definition 1.6 Assume $Q$ is a locally finite poset with a unique minimum element $\hat{0}$. We define the augmented Tchebyshev transform $\breve{T}(Q)$ of $Q$ as $T(Q \cup\{\widehat{-1}\})$, where $\widehat{-1}$ is a new minimum element, covered only by $\hat{0}$.

As a consequence of Proposition 1.3, the element $(\widehat{-1}, \hat{0}) \in \check{T}(Q)$ is the unique minimum element of $\check{T}(Q)$. In this paper we will also need the following statement, related to Lemma 1.4.

Lemma 1.7 Given $y_{1} \leq x_{2}<y_{2} \in Q$, and any pair of elements $x_{1}, x_{1}^{\prime} \in Q$ satisfying $x_{1}, x_{1}^{\prime}<y_{1}$, the intervals $\left[\left(x_{1}, y_{1}\right),\left(x_{2}, y_{2}\right)\right]$ and $\left[\left(x_{1}^{\prime}, y_{1}\right),\left(x_{2}, y_{2}\right)\right]($ in $T(Q)$ or $\check{T}(Q))$ are isomorphic.

Proof: Let us describe first the elements $(x, y)$ of $\left[\left(x_{1}, y_{1}\right),\left(x_{2}, y_{2}\right)\right]$. We may distinguish three disjoint cases, depending on whether $x=x_{1}, x=x_{2}$ or $x \notin\left\{x_{1}, x_{2}\right\}$. (The elements $x_{1}$ and $x_{2}$ are different, since $x_{1}<y_{1} \leq x_{2}$.) If $x=x_{1}$ then $\left(x_{1}, y_{1}\right) \leq(x, y)$ is equivalent to $y_{1} \leq y$, while $(x, y) \leq\left(x_{2}, y_{2}\right)$ is equivalent to $y \leq x_{2}$. If $x=x_{2}$ then $\left(x_{1}, y_{1}\right) \leq(x, y)$ is automatically satisfied, while $(x, y) \leq\left(x_{2}, y_{2}\right)$ is equivalent to $y \leq y_{2}$. Finally if $x$ is 
different from $x_{1}$ and $x_{2}$ then $\left(x_{1}, y_{1}\right) \leq(x, y) \leq\left(x_{2}, y_{2}\right)$ is equivalent to $y_{1} \leq x<y \leq x_{2}$. To summarize, we obtain a disjoint union description

$$
\begin{aligned}
{\left[\left(x_{1}, y_{1}\right),\left(x_{2}, y_{2}\right)\right]=} & \left\{\left(x_{1}, y\right): y_{1} \leq y \leq x_{2}\right\} \uplus\left\{\left(x_{2}, y\right): x_{2}<y \leq y_{2}\right\} \\
& \uplus\left\{(x, y): y_{1} \leq x<y \leq x_{2}\right\} .
\end{aligned}
$$

A similar formula may be written for $\left[\left(x_{1}^{\prime}, y_{1}\right),\left(x_{2}, y_{2}\right)\right]$. It may be observed immediately that removing the elements of the form $\left(x_{1}, y\right)$ from $\left[\left(x_{1}, y_{1}\right),\left(x_{2}, y_{2}\right)\right]$ yields the same set as removing the elements of the form $\left(x_{1}^{\prime}, y\right)$ from $\left[\left(x_{1}^{\prime}, y_{1}\right),\left(x_{2}, y_{2}\right)\right]$. Thus the map $\kappa:\left[\left(x_{1}, y_{1}\right),\left(x_{2}, y_{2}\right)\right] \rightarrow\left[\left(x_{1}^{\prime}, y_{1}\right),\left(x_{2}, y_{2}\right)\right]$ given by

$$
\kappa(x, y)= \begin{cases}(x, y) & \text { if } x>x_{1} \\ \left(x_{1}^{\prime}, y\right) & \text { if } x=x_{1}\end{cases}
$$

is a bijection. We only need to verify that it is also order-preserving. For that purpose let us compare each element of the form $\left(x_{1}, y\right)$ with the other elements in $\left[\left(x_{1}, y_{1}\right),\left(x_{2}, y_{2}\right)\right]$. Given another element $\left(x_{1}, y^{\prime}\right)$ of the same form, we have $\left(x_{1}, y\right) \leq\left(x_{1}, y^{\prime}\right)$ if and only if $y \leq y^{\prime}$. The actual value of $x_{1}$ is irrelevant for the purposes of this comparison. Since any element $\left(x_{1}, y\right) \in\left[\left(x_{1}, y_{1}\right),\left(x_{2}, y_{2}\right)\right]$ satisfies $y \leq x_{2}$, it is automatically less than any element of the form $\left(x_{2}, y^{\prime}\right)$ in $\left[\left(x_{1}, y_{1}\right),\left(x_{2}, y_{2}\right)\right]$. Given finally an element of the form $\left(x_{1}, y\right)$ and an element of the form $(x, y)$ where $x_{1}<x \neq x_{2}$, only $\left(x_{1}, y\right) \leq\left(x, y^{\prime}\right)$ is possible, if they are comparable at all, and the inequality holds if and only if $y \leq x$. This comparison is again independent of the actual value of $x_{1}$. Therefore $\kappa$ is indeed order preserving, since replacing $x_{1}$ with $x_{1}^{\prime}$ does not change any of the comparisons we need to make.

The original motivation behind the notion of the Tchebyshev transform in [6] was to introduce a sequence of Eulerian posets whose order complex encodes the Tchebyshev polynomials of the first kind.

Definition 1.8 Given any partially ordered set $P$, the order complex $\triangle(P)$ of $P$ is the simplicial complex whose vertices are the elements of $P$ and whose chains are the faces of $P$.

As noted at the end of Section 9 of [6], we have the following description of Tchebyshev polynomials.

Proposition 1.9 The Tchebyshev polynomial $T_{n}(x)$ of the first kind satisfies

$$
T_{n}(x)=\sum_{j=0}^{n} f_{j-1}(\triangle(((\widehat{-1}, \hat{0}),(-n,-(n+1))))) \cdot\left(\frac{x-1}{2}\right)^{j}
$$

where $((\widehat{-1}, \hat{0}),(-n,-(n+1)))$ is an open interval in the augmented Tchebyshev transform of the partially ordered set shown in figure 1. 
As usual, $f_{j-1}(\triangle)$ denotes the number of $j$-dimensional faces of a simplicial complex (which is also the number of $j$-element chains for an order complex). It was shown in [6, Theorem $4.1]$ that $\triangle(((\widehat{-1}, \hat{0}),(-n,-(n+1))))$ triangulates the boundary of the $n$-dimensional crosspolytope. This result may be generalized to intervals in an arbitrary Tchebyshev transform. To state the generalization, recall that the suspension $\Sigma(\triangle)$ of a simplicial complex is obtained by adjoining two new vertices, say $s_{1}$ and $s_{2}$, and adding the family $\left\{\left\{s_{1}\right\} \cup\right.$ $\left.\sigma,\left\{s_{2}\right\} \cup \sigma: \sigma \in \triangle\right\}$ to the set of faces. Moreover, given two simplicial complexes $\triangle_{1}$ and $\triangle_{2}$ with disjoint vertex sets, the join $\triangle_{1} * \triangle_{2}$ is defined as the simplicial complex $\triangle_{1} * \triangle_{2}=\left\{\sigma_{1} \cup \sigma_{2}: \sigma_{1} \in \triangle_{1}, \sigma_{2} \in \triangle_{2}\right\}$.

Theorem 1.10 Let $Q$ be a locally finite poset and consider an open interval $\left(\left(x_{1}, y_{1}\right)\right.$, $\left.\left(x_{2}, y_{2}\right)\right) \subset \check{T}(Q)$.

(i) If $x_{1}=x_{2}$ then $\triangle\left(\left(\left(x_{1}, y_{1}\right),\left(x_{2}, y_{2}\right)\right)\right)$ is isomorphic to $\triangle\left(\left(y_{1}, y_{2}\right)\right) \subset \Delta(Q)$.

(ii) If $x_{1} \neq x_{2}$ (and so $\left.y_{1} \leq x_{2}\right)$ then $\triangle\left(\left(\left(x_{1}, y_{1}\right),\left(x_{2}, y_{2}\right)\right)\right)$ is isomorphic to a triangulation of $\Sigma\left(\triangle\left(\left(y_{1}, x_{2}\right)\right) * \triangle\left(\left(x_{2}, y_{2}\right)\right)\right)$, where $\left(y_{1}, x_{2}\right)$ and $\left(x_{2}, y_{2}\right)$ are open intervals in $Q$.

Theorem 1.10 will only be used as a "source of inspiration" in this paper, its proof is outlined in the Appendix. Its significance in an algebraic setting is due to the fact that the Möbius function of an interval $[x, y]$ is the reduced Euler characteristic of $\triangle([x, y])$. (see [15, Proposition 3.8.6].) Hence Proposition 1.3 is a consequence of Theorem 1.10 and, more generally, we can expect the Möbius function to "behave nicely" on the intervals of some $\breve{T}(Q)$ if it "behaves nicely" on the intervals of $Q$.

\subsection{Noncommutative formal power series}

Given an alphabet $X$ of variables, the set of finite words with letters from $X$ (or, in other words, the free monoid generated by $X$ ) is usually denoted by $X^{*}$. Given a field $K$, a formal power series on $X$ is a formal linear combination $f=\sum_{w \in X^{*}} a_{w} w$ where all $a_{w}$ 's belong to $K$. Given a second formal power series $g=\sum_{w \in X^{*}} b_{w} w$, the sum of the two formal power series is defined by $f+g=\sum_{w \in X^{*}}\left(a_{w}+b_{w}\right) w$, while their product is defined as $f \cdot g=\sum_{w \in X^{*}}\left(\sum_{u v=w} a_{u} b_{v}\right) w$. The ring of formal power series on the alphabet $X$ with coefficient field $K$ is denoted by $K\langle\langle X\rangle\rangle$. Some information on noncommutative formal power series may be found in [16, Section 6.5].

For the purposes of our paper the following "typically noncommutative" phenomenon needs to be noted. For noncommutative formal power series there is often a distinct difference between factoring by an ideal generated by a single element, and the way someone used to commutative formal power series would tend to think "modulo the ideal". If, for example, one takes the ring $K[[x, y]]$ of formal power series in two commuting variables, then factoring by the ideal generated by $y$ is equivalent to removing all terms that contain a positive power of $y$, from all expressions. The factor ring is isomorphic to $K[[x]]$. In the noncommutative case, however, the formal power series $\sum_{n>0} x^{n} y x^{n}$ does not belong to the ideal generated by $y$. This is stated in Lemma 1.2 of the paper [4] by Gerritzen and Holtkamp. If we want to get a factor ring isomorphic to $K\langle\langle x\rangle\rangle=K[[x]]$, we need to factor 
by the ideal

$$
\bigcap_{n \geq 0}\left((y)+J^{n}\right),
$$

where $J^{n}$ is the ideal generated by all monomials of degree $n$. In general, given an ideal $I$ of $K\langle\langle X\rangle\rangle$, the closure of $I$ is the ideal

$$
\mathbf{c l}(I)=\bigcap_{n \geq 0}\left(I+J^{n}\right) .
$$

The reason for this terminology is the following. Consider the noncommutative polynomial ring $K\langle X\rangle$. Let us denote (by abuse of notation) also by $J$ the ideal generated by $X$ in $K\langle X\rangle$. Given any $p \in K\langle X\rangle$, the family of sets $\left\{p+J^{n}: n \in \mathbb{N}\right\}$ serves as the neighborhood basis in the $J$-adic topology on $K\langle X\rangle$. The noncommutative power series ring $K\langle\langle X\rangle\rangle$ is then the completion of $K\langle X\rangle$ with respect to this topology. (see e.g. [4, Section 1].) According to [4, Lemma 1.1] an ideal $I$ of $K\langle\langle X\rangle\rangle$ is closed in the $J$-adic topology if and only if

$$
I=\bigcap_{n \geq 0}\left(I+J^{n}\right) .
$$

The quotient by the closure of an ideal often corresponds better to the kind of quotient ring we grew used to in the commutative case.

\section{The augmented Tchebyshev transform of a binomial poset}

Since any binomial poset $Q$ is assumed to have a unique minimum element $\hat{0}$, it makes sense to consider its augmented Tchebyshev transform, which has a unique minimum element $(\widehat{-1}, \hat{0})$. Moreover, if any locally finite poset $Q$ has a rank function satisfying $\rho(\hat{0})=0$, then we may extend it to $Q \cup\{\widehat{-1}\}$ by setting $\rho(\widehat{-1})=-1$, and so by Proposition 1.3 we obtain that $\check{T}(Q)$ has a rank function given by $\rho(x, y)=\rho(y)$. Note that for this rank function the rank of the minimum element $(\widehat{-1}, \hat{0}) \in \breve{T}(Q)$ is zero.

Unfortunately, the augmented Tchebyshev transform of a binomial poset is not binomial even in the case of the simplest possible example. If $Q$ is a binomial poset then, by what was said above, $\check{T}(Q)$ satisfies all criteria of a binomial poset, except for the one requiring that the number of saturated chains of an interval has to depend on the rank of the interval only.

Example 2.1 Consider the binomial poset $\mathbb{N}$ consisting of all natural numbers and the usual linear order $0<1<2<\ldots$ on them. This is a binomial poset, with the "simplest possible" factorial function given by $B(n)=1$ for all $n \geq 0$. All elements below $(3,4)$ of the augmented Tchebyshev transform $\check{T}(\mathbb{N})$ are represented in figure 2 .

The intervals $[(-1,0),(1,3)]$ and $[(-1,0),(2,3)]$ both have rank 3 , but the number of saturated chains in the first interval is 2 , while in the second it is 4 . 


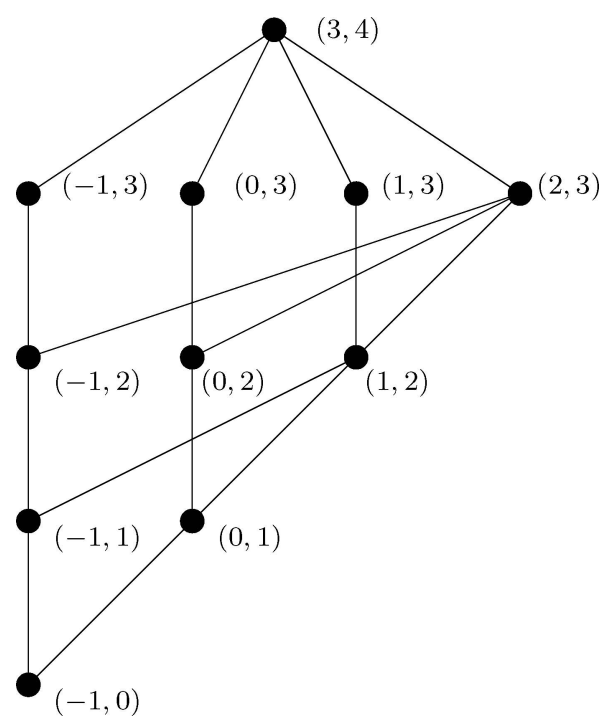

Figure 2. The interval $[(-1,0),(3,4)]$ in $\breve{T}(\mathbb{N})$.

We should not be discouraged by this example, because we only need to refine the picture a little bit to arrive at a situation reminiscent of the one of binomial posets.

Definition 2.2 Let $Q$ be a locally finite partially ordered set that has a minimum element $\hat{0}$ and a rank function $\rho$. Assume $\rho(\hat{0})=0$ and extend the rank function to $Q \cup\{\widehat{-1}\}$ by setting $\rho(\widehat{-1})=-1$. We call the ordered pair $(\rho(x), \rho(y))$ associated to $(x, y) \in \check{T}(Q)$ the type of $(x, y) \in \check{T}(Q)$.

As we will see in a moment, the number of saturated chains in an interval $[(u, v),(x, y)] \subset$ $\check{T}(Q)$ depends only on the type of its endpoints.

Lemma 2.3 Assume that $(u, v)<(x, y)$ in $\check{T}(Q)$, where $Q$ is any locally finite poset having a minimum element $\hat{0}$ and a rank function $\rho$.

1. If $u<x$ (and so $v \leq x$ ) then every saturated chain of $[(u, v),(x, y)] \subset \check{T}(Q)$ may be uniquely described by a pair of the following two objects:

(i) a saturated chain $v=y_{0} \prec y_{1} \prec \cdots \prec y_{\rho(y)-\rho(v)}=y$ of $[v, y] \subseteq Q$ satisfying $y_{\rho(x)-\rho(v)}=x$, and

(ii) A word $w_{1} w_{2} \ldots w_{\rho(y)-\rho(v)}$ formed of the letters $R$ and $L$ such that $w_{\rho(x)-\rho(v)+1}=L$ and all subsequent letters are $R$ 's.

2. If $u=x$ then the saturated chains of $[(u, v),(u, y)] \subset \check{T}(Q)$ are in bijection with the saturated chains of $[v, y] \subseteq Q$. 
Proof: This lemma is an almost straightforward consequence of Proposition 1.2. Assume $(u, v)<(x, y)$ and $u<x$ first. Consider an arbitrary saturated chain

$$
(u, v)=\left(x_{0}, y_{0}\right) \prec\left(x_{1}, y_{1}\right) \prec \cdots \prec\left(x_{\rho(y)-\rho(v)}, y_{\rho(y)-\rho(v)}\right)=(x, y)
$$

in $[(u, v),(x, y)]$. By Proposition 1.2, the element $\left(x_{i+1}, y_{i+1}\right)$ covers $\left(x_{i}, y_{i}\right)$ exactly when $y_{i+1}$ covers $y_{i}$, and $x_{i+1}$ is either $x_{i}$ or $y_{i}$. Thus the sequence $v=y_{0} \prec y_{1} \prec \cdots \prec$ $y_{\rho(y)-\rho(v)}=y$ must be a saturated chain in $[v, y] \subseteq Q$. Once such a saturated chain is fixed, we have at most two choices when we move from $\left(x_{i}, y_{i}\right)$ to $\left(x_{i+1}, y_{i+1}\right)$ : either we "remove the left element $x_{i}$ " and write $y_{i+1}$ after $y_{i}$ (yielding $\left.\left(x_{i+1}, y_{i+1}\right)=\left(y_{i}, y_{i+1}\right)\right)$, or we "remove the right element $y_{i}$ " and replace that with $y_{i+1}$ (yielding $\left(x_{i+1}, y_{i+1}\right)=\left(x_{i}, y_{i+1}\right)$ ). Let us record the first choice by a letter $L$ and the second choice by a letter $R .{ }^{1}$ For example, the saturated chain $0 \prec 1 \prec 2 \prec 3 \prec 4$ in $[0,4] \subset \mathbb{N}$ and the word $L R R L$ yields the saturated chain

$$
(-1,0) \prec(0,1) \prec(0,2) \prec(0,3) \prec(3,4)
$$

for the interval represented in figure 2. No matter which letter we choose, the rank of the second coordinate (which is also the rank of the element in $\check{T}(Q)$ ) always increases by one. The effect of the letters $L$ and $R$ on the rank of the first coordinate is completely different. Writing $R$ keeps the rank of the first coordinate unchanged, while writing $L$ increases the rank of the first coordinate to the rank of the second coordinate of the input. Hence, once the rank of the first coordinate reaches $\rho(x)$, we may use only $R$ 's, keeping the first coordinate unchanged. Thus we are only allowed to use $R$ 's once $x$ is introduced as a first coordinate for the first time. This first introduction of $x$ as a first coordinate can be only achieved by choosing the $L$-option which makes the second coordinate of the previous $\left(x_{i}, y_{i}\right)$ equal to $x$. The only $y_{i}$ that has the same rank as $x$ is $y_{\rho(x)-\rho(v)}$. Therefore we must have $y_{\rho(x)-\rho(v)}=x$, $w_{\rho(x)-\rho(v)+1}=L$ and all subsequent letters must be $R$ 's. Conversely, any saturated chain of $[v, y]$ and any $L R$ word satisfying conditions (i) and (ii) yields a saturated chain

$$
(u, v)=\left(x_{0}, y_{0}\right) \prec\left(x_{1}, y_{1}\right) \prec \cdots \prec\left(x_{\rho(y)-\rho(v)}, y_{\rho(y)-\rho(v)}\right)
$$

for which the first coordinates stabilize at $x_{\rho(x)-\rho(v)+1}=x_{\rho(x)-\rho(v)+2}=\cdots=x$ and the second coordinates halt at $y_{\rho(y)-\rho(v)}=y$.

The proof for the case when $u=x$ is similar but easier. Here $x$ is already introduced as the first coordinate at the beginning of the saturated chain, so only the option represented by the letter $R$ may be used all along. Therefore the saturated chains of $[(u, v),(u, y)] \subset \breve{T}(Q)$ are in bijection of with the saturated chains of $[v, y] \subseteq Q$.

Corollary 2.4 Let $Q$ be a binomial poset with factorial function $B(n)$ and assume $(u, v) \leq$ $(x, y)$ in $\breve{T}(Q)$. If the type of $(u, v)$ is $(i, j)$ and the type of $(x, y)$ is $(k, l)$ then the number of saturated chains in $[(u, v),(x, y)]$ is $2^{k-j} B(k-j) B(l-k)$ if $i<k$, and $B(l-j)$ if $i=k$.

In fact, $i=k$ is only possible if $u=x$ and in that case $B(l-j)$ is the number of saturated chains in $[v, y]$. If $i<k$ then we are in the case $u<x$, the product $B(k-j) B(l-k)$ is the 
number of pairs of saturated chains, one in $[v, x]$, one in $[x, y]$, while $2^{k-j}$ is the number of admissible $L R$-words.

It is worth noting that in the case when $k \geq j$ the number of saturated chains from an element of type $(i, j)$ to an element $(k, l)$ is the same as the number of saturated chains from $(\widehat{-1}, \hat{0})(=$ the only element of type $(-1,0))$ to an element of type $(k-j, l-j)$. The only obstacle to automatically extending this observation to the case $k=i$ is that in that case we might have $k-j<-1$ and no element of $Q \cup\{\widehat{-1}\}$ has such a low rank. This motivates the following definition.

Definition 2.5 We define the type of an interval $[(u, v),(x, y)] \subset \check{T}(Q)$ to be

$$
(\max (\rho(x)-\rho(v),-1), \rho(y)-\rho(v)) .
$$

In analogy to binomial posets we define a factorial function on the types of intervals. Our factorial function will differ from the actual number of saturated chains for most types by a factor of a power of 2. The reason of this choice will be clear in Section 3 where we use our factorial function to express generalized binomial coefficients.

Definition 2.6 Let $Q$ be a binomial poset and $B$ its factorial function. For any pair of integers $(k, l)$ satisfying $k<l$ and $l \geq 0$ we define the factorial $B(k, l)$ associated to $\check{T}(Q)$ by the following formula.

$$
B(k, l)= \begin{cases}B(k) B(l-k) & \text { if } k \geq 0 \\ B(l) & \text { if } k<0\end{cases}
$$

Remark 2.7 If $(k, l)$ is the type of an interval, then we require $k \geq-1$, while $B(k, l)$ is defined for all negative values of $k$, although it always yields the same number as $B(-1, l)$. When we perform calculations with these coefficients, it will be more convenient to allow all values of $k$, while the number of saturated chains in an interval $[(u, v),(x, y)] \subset \check{T}(Q)$ satisfying $\rho(x)-\rho(v)<0$ (and thus $x=u$ ) does not depend on the actual value of $\rho(x)-\rho(v)$. Alternatively one could define the type of an interval $[(u, v),(x, y)] \subset \breve{T}(Q)$ to be $(\rho(x)-\rho(v), \rho(y)-\rho(v))$ and then declare that "all types $(k, l)$ with a fixed positive $l$ and an arbitrary negative $k$ are the same". This convention is also useful to state the following straightforward observation more easily: if $[(u, v),(x, y)]$ has type $(k, l)$ and $(r, s) \in[(u, v),(x, y)]$ is such that the type of $[(u, v),(r, s)]$ is $(m, n)$ then the type of $[(r, s),(x, y)]$ is $(k-n, l-n)$. Without the informal convention one would need to say that the type of $[(r, s),(x, y)]$ is $(\max (-1, k-n), l-n)$.

Using Definitions 2.5 and 2.6 we may restate Corollary 2.4 as follows.

Proposition 2.8 The number of saturated chains of an interval of type $(k, l)$ in $\check{T}(Q)$ is $2^{\max (0, k)} B(k, l)$. 


\section{Generalizing binomial coefficients}

As seen in the previous section, in the augmented Tchebyshev transform of a binomial poset, the number of saturated chains in an interval depends not only on the rank, but also on the type of the interval. Hence, rather than counting all elements of a given rank in an interval, it seems to make more sense to count all elements of a given type. Let us introduce $\left(\begin{array}{c}(i, j),(k, l) \\ (m, n)\end{array}\right)$ for the number of elements of type $(m, n)$ in an interval whose minimum element has type $(i, j)$ and maximum element has type $(k, l)$. Our main result is the following:

Proposition 3.1 Let $Q$ be a binomial poset. Then the binomial coefficients associated to $\check{T}(Q)$ satisfy the formula

$$
\left(\begin{array}{c}
(i, j),(k, l) \\
(m, n)
\end{array}\right)=\frac{B(k-j, l-j)}{B(m-j, n-j) B(k-n, l-n)}
$$

In particular, the number of elements $(r, s)$ of a fixed type in an interval $[(u, v),(x, y)]$ depends only on the type of the intervals $[(u, v),(x, y)]$ and $[(u, v),(r, s)]$.

Proof: Assume we are given an interval $[(u, v),(x, y)] \subseteq \check{T}(Q)$ such that $(u, v)$ is of type $(i, j)$ and $(x, y)$ is of type $(k, l)$. We want to count the number of elements $(r, s)$ of type $(m, n)$. By the definition of the Tchebyshev order, $(u, v) \leq(r, s)$ implies that either $u=r$ or $v \leq r$ must hold. At the level of the types we must either have $i=m$ or $j \leq m$. Similarly, from $(r, s) \leq(x, y)$ we have either $m=k$ or $n \leq k$, and from $(u, v) \leq(x, y)$ we have either $i \leq k$ or else $i<k$ and $j \leq k$.

Case 1: $i=k$. In this case $m=i=k$ and also $u=r=x$ must hold. By the second case of Lemma 2.3, the saturated chains in $[(u, v),(x, y)]$ are then in bijection with the saturated chains of $[v, y]$. A saturated chain of $[(u, v),(x, y)]$ contains $(r, s)$ if and only if the corresponding saturating chain of $[v, y]$ contains $s$. Hence we are reduced to count the number of elements of given rank in a binomial poset. Using the well known formula from Stanley's book [15, Section 3.15, Eq. (47)], we obtain

$$
\left(\begin{array}{c}
(i, j),(i, l) \\
(i, n)
\end{array}\right)=\frac{B(l-j)}{B(n-j) B(l-n)} .
$$

Case 2: $i<k$ (and so $j \leq k$ ). Now we are in the first case of Lemma 2.3. As seen there, every saturated chain of $[(u, v),(x, y)]$ may be described by a saturated chain

$$
v=y_{0} \prec y_{1} \prec \cdots \prec y_{l-j}=y
$$

of $[v, y]$ satisfying $y_{k-j}=x$, and an $L R$-word $w_{1} w_{2} \ldots w_{l-j}$ such that $w_{k-j+1}=L$ and all subsequent letters are $R$ 's. 
Assume first $j \leq m<n \leq k$, the other subcases being similar but easier. To decide whether the saturated chain of $[(u, v),(x, y)]$ contains any element of type $(m, n)$, one only needs to know the associated $L R$ word. In fact, some element of rank $m$ is introduced as a first coordinate if and only if $w_{m-j+1}=L$. This element is still the first coordinate when the second element reaches rank $n$ if and only if $w_{m-j+1}$ is followed by $n-m-1$ $R$ 's. As a consequence, every element of type $(m, n)$ is contained in the same number of saturated chains of $[(u, v),(x, y)]$ (associated to the same set of $L R$-words). The number of elements of type $(m, n)$ equals the total number of saturated chains containing some element of type $(m, n)$ divided by the number of saturated chains containing a fixed element of type $(m, n)$. (This part of our reasoning is analogous to the classical case.) When we perform this division, the number of admissible $L R$-words cancels, and we are left with dividing the total number of saturated chains

$$
v=y_{0} \prec y_{1} \prec \cdots \prec y_{l-j}=y
$$

satisfying $y_{k-j}=x$ with the number of similar saturated chains also satisfying $y_{m-j}=r$ and $y_{n-j}=s$, where $(r, s) \in \breve{T}(Q)$ is an arbitrary but fixed element of type $(m, n)$. Therefore we have

$$
\begin{aligned}
\left(\begin{array}{c}
(i, j),(k, l) \\
(m, n)
\end{array}\right) & =\frac{B(k-j) \underline{B}(l-k)}{B(m-j) B(n-m) B(k-n) \underline{B}(l-k)} \\
& =\frac{B(k-j)}{B(m-j) B(n-m) B(k-n)} .
\end{aligned}
$$

The remaining subcases are $m=i$ and $m=k$. Similarly to the previous subcase, the number of admissible $L R$ words cancels when we divide the total number of saturated chains containing some element of the prescribed type with the number of saturated chains containing an arbitrary but fixed element of the prescribed type. In the subcase when $m=i$ (and so $m=i<j \leq n \leq k<l$ ), we have to divide the number of saturated chains

$$
v=y_{0} \prec y_{1} \prec \cdots \prec y_{l-j}=y
$$

satisfying $y_{k-j}=x$ with the number of similar saturated chains also satisfying $y_{n-j}=s$ where $s \in Q$ is an arbitrary but fixed element of rank $n$. Therefore we have

$$
\left(\begin{array}{c}
(i, j),(k, l) \\
(i, n)
\end{array}\right)=\frac{B(k-j) \underline{B(l-k)}}{B(n-j) B(k-n) \underline{B}(l-k)}=\frac{B(k-j)}{B(n-j) B(k-n)} .
$$

Finally, when $m=k$ (and so $i<j \leq k=m<n \leq l$ ) the same division as in the previous subcase yields

$$
\left(\begin{array}{c}
(i, j),(k, l) \\
(k, n)
\end{array}\right)==\frac{\underline{B(k-J) B(l-k)}}{\underline{B(k-J) B(n-k) B(l-n)}}=\frac{B(l-k)}{B(n-k) B(l-n)} .
$$


The four equations we obtained for $\underset{(m, n)}{(i, j),(k, l)}$ ) depending on the relation between $m$ and the other entries, look rather different. Ironically this is due to writing our binomial coefficient in simplest form in terms of the factorial function of $Q$. If we use the factorial function introduced in Definition 2.6, the formula stated in the Proposition follows from Eqs. (2), (3), (4), and (5) by straightforward substitution.

Corollary 3.2 Assume that $[(u, v),(x, y)] \subset \check{T}(Q)$ has type $(k, l)$. Then the number of those elements $(r, s) \in[(u, v),(x, y)]$ for which the type of $[(u, v),(r, s)]$ is $(m, n)$ is

$$
\left(\begin{array}{c}
(-1,0),(k, l) \\
(m, n)
\end{array}\right)=\frac{B(k, l)}{B(m, n) B(k-n, l-n)} .
$$

This corollary is an immediate consequence of Proposition 3.1 and Remark 2.7.

\section{Generating functions}

In analogy with the theory built for binomial posets, consider the following subalgebra of the incidence algebra of $\breve{T}(Q)$

Definition 4.1 Given a binomial poset $Q$ and a field $K$, we say that a function $f \in$ $I(\breve{T}(Q), K)$ is a function of types if it assigns the same value to all intervals of the same type. We denote the subalgebra of the functions of types by $R(\check{T}(Q), K)$.

Remark 4.2 Our notation looks similar to the one introduced in Stanley's book [15, Section 3.15] for binomial posets and a different set of functions. However, this will not lead to confusion, since for any locally finite poset $Q$ with a unique minimum element $\hat{0}$, its augmented Tchebyshev transform $\breve{T}(Q)$ is never binomial. Assume the contrary. Applying Lemma 1.4 to all intervals of the form $[(\widehat{-1}, x),(\widehat{-1}, y)] \subseteq \breve{T}(Q)$ we may easily convince ourselves that $Q$ must be binomial. Using the rank function of $Q$ we may define the types of the intervals in $\breve{T}(Q)$, and observe that all intervals of type $(k, l)$ with a fixed $l$ must have the same number of saturated chains. In particular, we must have that the number of saturated chains is the same in an interval of type $(1,3)$ and in an interval of type $(2,3)$. By Proposition 2 this is equivalent to $2 B(1) B(2)=4 B(2) B(1)$, which can never happen, considering that $B(1)$ and $B(2)$ must be positive.

By abuse of notation, for a function of types $f \in R(\check{T}(Q), K)$ we will denote its value on an arbitrary interval of type $(k, l)$ by $f(k, l)$. Furthermore, for the sake of notational convenience we extend the definition of $f(k, l)$ to all negative integers $k$ by setting $f(k, l)=$ $f(-1, l)$ for all $k<0$. Using this notational convenience, and keeping in mind Remark 2.7, we may write the following "convolution formula":

$$
(f \cdot g)(k, l)=\sum_{(-1,0) \leq(m, n) \leq(k, l)}\left(\begin{array}{c}
(-1,0),(k, l) \\
(m, n)
\end{array}\right) f(m, n) \cdot g(k-n, l-n)
$$


Observe that the partial order below the summation sign is exactly the partial order of $\breve{T}(\mathbb{N})$ introduced in Example 2.1. According to Corollary 3.2 this equation may be rewritten as

$$
(f \cdot g)(k, l)=\sum_{(-1,0) \leq(m, n) \leq(k, l)} \frac{B(k, l)}{B(m, n) B(k-n, l-n)} f(m, n) \cdot g(k-n, l-n)
$$

or, equivalently

$$
\frac{(f \cdot g)(k, l)}{B(k, l)}=\sum_{(-1,0) \leq(m, n) \leq(k, l)} \frac{f(m, n)}{B(m, n)} \cdot \frac{g(k-n, l-n)}{B(k-n, l-n)} .
$$

In analogy to the case of binomial posets, the existence of these convolution rules demonstrates the fact that $R(\check{T}(Q), K)$ is indeed a subalgebra of $I(\check{T}(Q), K)$. Rule (6) suggests considering generating functions of the form

$$
\phi(f)=\sum_{-1 \leq k<l<\infty} \frac{f(k, l)}{B(k, l)} \cdot x(k, l)
$$

where the multiplication rules for the "monomials" $x(k, l)$ have to be deciphered from equation (6).

Definition 4.3 Given a field $K$ we define the Tchebyshev algebra $T(K)$ of $K$ as the algebra of infinite formal sums

$$
\sum_{-1 \leq k<l<\infty} a_{k, l} \cdot x(k, l)
$$

where all coefficients $a_{k, l}$ belong to $K$, and the terms $x(k, l)$ obey the multiplication rule

$$
x\left(i_{1}, i_{2}\right) \cdot x\left(j_{1}, j_{2}\right)= \begin{cases}x\left(i_{2}+j_{1}, i_{2}+j_{2}\right) & \text { if } j_{1} \geq 0 \\ x\left(i_{1}, i_{2}+j_{2}\right) & \text { if } j_{1}<0\end{cases}
$$

Before going any further let us observe that by setting $\operatorname{deg}(x(k, l))=l$ we may define a "degree function" on the terms, and there are only finitely many values of $k$ satisfying $-1 \leq k<l$ for a fixed value of $l$. Hence the multiplication rule given in the definition induces a valid multiplication rule for products of infinite sums, since there will be only finitely many terms contributing to the coefficient of any given $x(k, l)$ in the product. In particular, we have the following.

Proposition 4.4 Let $f=\sum_{-1 \leq k<l} a_{k, l} x(k, l)$ and $g=\sum_{-1 \leq k<l} b_{k, l} x(k, l)$ be two elements of $T(K)$. Extend the definition of $b_{k, l}$ to all negative $k$ 's by setting $b_{k, l}=b_{-1, l}$ 
whenever $k<0$. Then the coefficient of $x(k, l)$ in $f \cdot g$ is

$$
\sum_{(-1,0) \leq(m, n) \leq(k, l)} a_{m, n} \cdot b_{k-n, l-n}
$$

where the partial order below the summation sign is the partial order of $\breve{T}(\mathbb{N})$.

Proof: Let us fix $x(k, l)$ and $x(m, n)$. First we show that $x(m, n) \cdot x(i, j)=x(k, l)$ holds for some $x(i, j)$ exactly when $(m, n) \leq(k, l)$ and that such an $x(i, j)$ is unique.

Let us try setting $i=-1$ first. Then, by the multiplication rule we have

$$
x(m, n) \cdot x(-1, j)=x(m, n+j) .
$$

This is equal to $x(k, l)$ iff. $m=k$ and $j=l-n$. The requirement $j>-1$ is equivalent to $n \leq l$.

Consider now the case $i \geq 0$. Then, the definition yields

$$
x(m, n) \cdot x(i, j)=x(n+i, n+j) .
$$

This is equal to $x(k, l)$ iff. $i=k-n$ and $j=l-n$. Since $i$ is not negative, we must have $n \geq k$. The requirement $j>i$ is an automatic consequence of $l>k$.

We obtained that $x(m, n) \cdot x(i, j)=x(k, l)$ has a solution exactly when either both $m=k$ and $n \leq l$ hold, or when $k \geq n$. This is exactly the definition of the partial order $\check{T}(\mathbb{N})$ for $(m, n)$ and $(k, l)$. In each case we have found a unique solution. In the second case we found this unique solution to be $x(k-n, l-n)$, in the first case we found $x(-1, l-n)$. In this first case $k-n$ is negative and we have set $b_{k-n, l-n}=b_{-1, l-n}$. This observation concludes the rest of the proof.

Equation (6) and Proposition 4.4 yield the following theorem.

Theorem 4.5 Given any binomial poset $Q$, the function $\phi: R(\check{T}(Q), K) \rightarrow T(K)$ defined by

$$
\phi(f)=\sum_{-1 \leq k<l<\infty} \frac{f(k, l)}{B(k, l)} \cdot x(k, l)
$$

is an algebra-isomorphism.

The proof is analogous to the proof of [15, Theorem 3.15.4]. We conclude this section with a few observations about the Tchebyshev algebra.

Corollary 4.6 The algebra $T(K)$ is associative. 
In fact, by Theorem 4.5, $T(K)$ is isomorphic to a subalgebra of the incidence algebra of a partially ordered set. It is also easy to verify the associativity directly on the semigroup of monomials $x(k, l)$, from which general associativity directly follows.

Proposition $4.7 x(-1,0)$ is the multiplicative identity of $T(K)$.

This statement is straightforward. Using this observation we call $x(-1,0)$ and its coefficient the constant term of an element of $T(K)$.

Since the Tchebyshev algebra is obviously not commutative, there is a distinction between left and right inverses. Fortunately we have the following statement, in analogy to the case of formal power series.

Proposition 4.8 If $f \in T(K)$ has either a left or a right inverse then its constant term is nonzero. Conversely, if $f \in T(K)$ has a nonzero constant term, then it has both left and right inverses (which therefore must be equal).

Proof: If the constant term of $f$ is zero, then its lowest degree terms have positive degree. Hence the lowest degree terms in any product $f \cdot g$ or $g \cdot f$ will also have positive degrees. It is thus necessary for $f$ to have a nonzero constant term if it has any one-sided inverse.

To prove the converse, observe first that, by Proposition 4.4, given an $f=\sum_{(k, l)} a_{k, l}$ $x(k, l) \in T(K)$ such that $a_{-1,0} \neq 0$, a right inverse $g=\sum_{(k, l)} b_{k, l} x(k, l)$ may be found by setting $b_{-1,0}=1 / a_{-1,0}$ and solving

$$
0=\sum_{(-1,0) \leq(m, n) \leq(k, l)} a_{m, n} \cdot b_{k-n, l-n}
$$

for all $(k, l) \neq(-1,0)$. One may show by induction on $l$, that such a system of $b_{k, l}$ 's exist. In fact, the only place where $b_{k, l}$ occurs in the above equation is when $(m, n)=(-1,0)$. Hence we may write

$$
b_{k, l}=-\frac{1}{a_{-1,0}} \cdot \sum_{(-1,0)<(m, n) \leq(k, l)} a_{m, n} \cdot b_{k-n, l-n}
$$

where the second index of each $b_{i, j}$ on the right hand side is strictly less than $l$, and we only divide by the nonzero $a_{-1,0}$. The proof of the existence of a left inverse is similar, only easier. Both arguments are analogous to showing that the Möbius function is the inverse of the zeta function and reiterate the fact that an upper triangular matrix is invertible if and only if its diagonal entries are nonzero.

\section{Structure and representation of the Tchebyshev algebra}

Definition 4.3 was designed in a way that made it easy to prove that the Tchebyshev algebra provides generating functions for the functions on types of intervals in some $\check{T}(Q)$. This definition does not reveal, however, much of the structure of $T(K)$. 
Theorem 5.1 The Tchebyshev algebra $T(K)$ is isomorphic to the quotient of the noncommutative formal power series ring $K\langle\langle x, y\rangle\rangle$ by the closure of the ideal generated by $y x-x^{2}$. This isomorphism may be given by replacing each $x(-1, l)$ with $y^{l}$ and each $x(k, l)$ (where $k \geq 0$ ) with $x^{k+1} y^{l-k-1}$.

Proof: Let us show first that every element of the ring

$$
K\langle\langle x, y\rangle\rangle / \bigcap_{n \geq 0}\left(\left(y x-x^{2}\right)+J^{n}\right)=K\langle\langle x, y\rangle\rangle / \operatorname{cl}\left(y x-x^{2}\right)
$$

may be uniquely written as an infinite linear combination of noncommutative monomials of the form $x^{i} y^{j}$, where $i, j \geq 0$. Any monomial may be rearranged into the form $x^{i} y^{j}$ by the use of the rule $y x=x^{2}$ a finite number of times. There is only a finite number of noncommutative monomials that are congruent to the same $x^{i} y^{j}$ modulo $\left(y x-x^{2}\right)$ since we factor by a homogeneous ideal, and there are only finitely many noncommutative monomials of degree $i+j$. Thus any noncommutative polynomial of $x$ and $y$ is obviously congruent to a linear combination of $x^{i} y^{j}$,s modulo $\left(y x-x^{2}\right)$. Moreover, it is easy to see that different linear combinations of $x^{i} y^{j}$ 's are incongruent modulo $\left(y x-x^{2}\right)$. In fact, every nonzero element of the ideal $\left(y x-x^{2}\right)$ contains at least one monomial with nonzero coefficient in which a $y$ precedes an $x$, and so it cannot be the difference of linear combinations of $x^{i} y^{j}$ 's.

Consider now a noncommutative formal power series $f \in K\langle\langle x, y\rangle\rangle$ which may consist of infinitely many nonzero terms. Using the relation $y x=x^{2}$ to rearrange all monomials of degree at most $n$, we may write up a formal power series $f_{n}$ which is congruent to $f$ modulo $\left(y x-x^{2}\right)$, and up to degree $n$ it consists only of terms of the form $a_{i, j} x^{i} y^{j}$. Moreover, given $m<n$, the terms of $f_{m}$ and $f_{n}$ agree up to degree $m$. Hence there is a "limit" $g$ which agrees with every $f_{n}$ up to degree $n$. (As a matter of fact, $g$ is the limit of the series $f_{1}, f_{2}, \ldots$ in the $J$-adic topology.) In other words, $g-f_{n} \in\left(y x-x^{2}\right)+J^{n+1}$, and so $g-f=g-f_{n}+f_{n}-f \in\left(y x-x^{2}\right)+J^{n+1}$ holds for all $n$. Therefore $g$ is congruent to $f$ modulo the closure of $y x-x^{2}$ and it is obviously of the required form.

The uniqueness follows from the fact that for all $n$, the sum of the terms of $g$ up to degree $n$ forms a polynomial which is uniquely defined.

Let us denote $x(0,1)$ by $x$ and $x(-1,1)$ by $y$. It is easy to show by induction on $n$ that

$$
x^{n}=x(n-1, n) \quad \text { and } \quad y^{n}=x(-1, n)
$$

hold for all positive $n$. The formula is also valid for $n=0$ since $x(-1,0)$ is the identity element of $T(K)$. Thus we have

$$
y x=x(-1,1) x(0,1)=x(1,2)=x^{2}
$$

and for positive $i$ (and nonnegative $j$ ) we also have

$$
x^{i} y^{j}=x(i-1, i) x(-1, j)=x(i-1, i+j) .
$$

Therefore the unique solution to $x^{i} y^{j}=x(k, l)$ is $i=k+1$, and $j=l-k-1$. 
We have proved that $T(K)$ consists of infinite linear combinations of the exact same kind, with the exact same multiplication and addition rules as the ones holding in the factor of $K\langle\langle x, y\rangle\rangle$ by the closure of $\left(y x-x^{2}\right)$.

The isomorphism $\phi: R(\check{T}(Q), K) \rightarrow T(K)$ depends on the structure of the binomial poset $Q$. On the other hand, between $K\langle\langle x, y\rangle\rangle / \operatorname{cl}\left(y x-x^{2}\right)$ and $T(K)$ we will always consider the same isomorphism: the one that sends $x$ into $x(0,1)$ into $x$, and $x(-1,1)$ into $y$. Hence we will identify these two rings in this paper, and use the notations $x^{k+1} y^{l-k-1}$ and $x(k, l)$ interchangeably.

Remark 5.2 The ideal generated by $y x-x^{2}$ in $K\langle\langle x, y\rangle\rangle$ is not closed in the $J$-adic topology. This may be shown by proving that the noncommutative formal power series

$$
\sum_{k=0}^{\infty} x^{k}\left(y x-x^{2}\right) x^{k}
$$

does not belong to $\left(y x-x^{2}\right)$. According to Ralf Holtkamp [10], this statement may be shown in analogy to the proof of Lemma 1.2 in the paper [4] by Gerritzen and Holtkamp.

Remark 5.3 Multiplication by $x$ and $y$ in $T(K)$ may be easily "visualized" using a picture of $\breve{T}(\mathbb{N})$, such as the one represented in figure 2 . We may identify the monomial $x(i, j)$ with the element $(i, j) \in \check{T}(\mathbb{N})$ on the picture. Multiplying by $y=x(-1,1)$ corresponds then to moving straight up (such moves are indexed with the letter $R$ in the statement of Lemma 2.3), while multiplying by $x=x(0,1)$ corresponds to to moving to the rightmost element in the row right above (these are the moves indexed by $L$ ). It is also worth noting that, given any $(i, j) \in \check{T}(\mathbb{N})$, the upper ideal $\{(u, v):(u, v) \geq(i, j)\}$ is isomorphic to $\breve{T}(\mathbb{N})$, which "explains" why multiplication in $T(K)$ is associative. (The proof of this "self-similarity" is analogous to the proof of [6, Proposition 3.1], and left to the reader.)

Remark 5.4 The study of the Tchebyshev algebra provides not only an analogous theory to the study of formal power series associated to binomial posets, but it is a generalization of the classical theory. In fact, the map $\alpha: K\langle\langle x, y\rangle\rangle / \operatorname{cl}\left(y x-x^{2}\right) \rightarrow K[[t]]$ induced by $\alpha(x)=0$ and $\alpha(y)=t$ is an algebra homomorphism, which may be completed to the following commutative diagram:

$$
\begin{aligned}
R(\breve{T}(Q), K) & \cong \\
\alpha_{R} \downarrow & \downarrow\langle\langle x, y\rangle\rangle / \mathbf{c l}\left(y x-x^{2}\right) \\
R(Q, K) & \cong K[[t]]
\end{aligned}
$$

Here $\alpha_{R}: R(\check{T}(Q), K) \rightarrow R(Q, K)$ is given by $\alpha_{R}(f)(l)=f(-1, l)$. The fact that $\alpha_{R}$ is a homomorphism may be established using Lemma 1.4. In fact, exactly those intervals $[(x, y),(u, v)]$ of $\breve{T}(Q)$ have type $(-1, l)$ for some $l$ which satisfy $x=u$. According to Lemma 1.4 , the interval $[(x, y),(x, v)]$ is isomorphic to the interval $[y, v]$ of $Q$. The effect of $\alpha_{R}$ is thus to restrict the domain of $f$ to such intervals of $\breve{T}(Q)$ which are isomorphic 
to intervals of $Q$. Using this observation we may extend $\alpha_{R}$ to the homomorphism $\alpha_{I}$ : $I(\breve{T}(Q), K) \rightarrow I(Q, K)$ of incidence algebras, given by

$$
\alpha_{I}(f)([x, y])=f([(\widehat{-1}, x),(\widehat{-1}, y)]) .
$$

Using Lemma 1.4, one may show that $\alpha_{I}$ defined by equation (8) is a homomorphism from $I(\breve{T}(Q), K)$ onto $I(Q, K)$ even if $Q$ is not a binomial poset!

There are many ways to represent the Tchebyshev algebra as a ring of matrices of (commutative) formal power series in a single variable. In particular, we are about to show that there is a sequence of homomorphisms $\Phi_{d}: T(K) \rightarrow K[[t]]^{d \times d}$ such that the restriction of $\Phi_{d}$ to the to the linear span of monomials of degree at most $d-1$ is injective.

Before explicitly defining such a sequence of representations, let us make a few general observations. Assume that some homomorphism $\Phi: T(K) \rightarrow K[[t]]^{d \times d}$ sends $x$ into $X$ and $y$ into $Y$. Setting $X$ and $Y$ uniquely determines a homomorphism from the (noncommutative) polynomial ring $K\langle x, y\rangle$ into $K[[t]]^{d \times d}$. If our homomorphism is "continuous", in the sense that given any sequence of polynomials converging in the $J$-adic topology, all entries of their images converge in the $(t)$-adic topology of $K[[t]]$, then the homomorphism uniquely extends to a homomorphism from $K\langle\langle x, y\rangle\rangle$ to $K[[t]]^{d \times d}$. One way to achieve this is by choosing our matrices $X$ and $Y$ in such a way that all their entries have zero constant term. The kernel of this continuous homomorphism will contain the closure of the ideal generated by $y x-x^{2}$ if and only if the matrices $X$ and $Y$ satisfy $Y X-X^{2}=0$, that is $(Y-X) X=0$. To summarize:

Proposition 5.5 Let $X$ and $Y$ be $d \times d$ matrices with entries in $K[[t]]$ such that each entry of $X$ and $Y$ has zero constant term and $(Y-X) X=0$ is satisfied. Then there is a unique homomorphism

$$
\Phi: K\langle\langle x, y\rangle\rangle / \mathbf{c l}\left(y x-x^{2}\right) \rightarrow K[[t]]^{d \times d}
$$

satisfying $\Phi(x)=X$ and $\Phi(y)=Y$.

Using matrices $X$ and $Y$ whose entries have zero constant term is a sufficient but not a necessary condition to guarantee the continuity of the induced homomorphism $K\langle x, y\rangle \rightarrow$ $K[[t]]^{d \times d}$. Consider, for example, the representation induced by setting

$$
X=\left(\begin{array}{ll}
t & 1 \\
0 & 0
\end{array}\right) \text { and } Y=\left(\begin{array}{ll}
t & 1 \\
0 & t
\end{array}\right)
$$

These matrices obviously satisfy $Y X=X^{2}$. Straightforward induction shows that

$$
X^{n}=\left(\begin{array}{ll}
t^{n} & t^{n-1} \\
0 & 0
\end{array}\right), \quad \text { and } \quad Y^{n}=\left(\begin{array}{ll}
t^{n} & n \cdot t^{n-1} \\
0 & t^{n}
\end{array}\right)
$$


hold for $n \geq 0$. For any positive $i$ we obtain

$$
X^{i} Y^{j}=\left(\begin{array}{ll}
t^{i} & t^{i-1} \\
0 & 0
\end{array}\right)\left(\begin{array}{ll}
t^{j} & j \cdot t^{j-1} \\
0 & t^{j}
\end{array}\right)=\left(\begin{array}{ll}
t^{i+j} & (j+1) \cdot t^{i+j-1} \\
0 & 0
\end{array}\right)
$$

In particular $x(k, l)=x^{k+1} y^{l-k-1}$ is represented by

$$
X(k, l)=X^{k+1} Y^{l-k-1}=\left(\begin{array}{ll}
t^{l} & (l-k) t^{l-1} \\
0 & 0
\end{array}\right)
$$

when $k$ is positive, and by

$$
X(-1, l)=Y^{l}=\left(\begin{array}{ll}
t^{l} & l \cdot t^{l-1} \\
0 & t^{l}
\end{array}\right)
$$

when $k=-1$. Clearly, when $l$ converges to infinity, each entry in $X^{k+1} Y^{l-k-1}$ converges to zero in the $(t)$-adic topology of $K[[t]]$. Hence the homomorphism sending $x$ into $X$ and $y$ into $Y$ is continuous. As a consequence of Theorem 4.5 we obtain the following:

Corollary 5.6 The function $\tilde{\Phi}: R(\breve{T}(Q), K) \rightarrow K[[t]]^{2 \times 2}$ defined by

$$
\tilde{\Phi}(f)=\left(\begin{array}{cc}
\sum_{-1 \leq k<l<\infty} \frac{f(k, l)}{B(k, l)} \cdot t^{l} & \sum_{0 \leq l<\infty} \frac{f(-1, l)}{B(-1, l)} \cdot l \cdot t^{l-1}+\sum_{0 \leq k<l<\infty} \frac{f(k, l)}{B(k, l)} \cdot(l-k) \cdot t^{l-1} \\
0 & \sum_{0 \leq l<\infty} \frac{f(-1, l)}{B(-1, l)} \cdot t^{l}
\end{array}\right)
$$

is an algebra-homomorphism.

There are infinitely many ways to represent $R(\breve{T}(Q), K)$, and all of them may yield interesting formulas for matrices of formal power series. In this paper we focus on one sequence of representations

$$
\Phi_{d}: K\langle\langle x, y\rangle\rangle / \mathbf{c l}\left(y x-x^{2}\right) \rightarrow K[[t]]^{d \times d}
$$

$(d=1,2, \ldots)$ that has the additional property of $\Phi_{d}$ being injective on the linear span of all monomials of degree at most $d-1$. Thus the direct sum $\oplus_{d \geq 1} \Phi_{d}$ provides a faithful representation of the Tchebyshev algebra. (Actually, it will turn out that it will be possible to take the "limit" $\lim _{d \rightarrow \infty} \Phi_{d}$ of our homomorphisms and obtain a single representation by infinite lower triangular matrices.) 
To facilitate our calculations, let us fix $d$ and denote by $E_{i, j}$ the $d \times d$ matrix whose only nonzero entry is a 1 in the $i$-th row and $j$-th column. Let us set

$$
X=t \cdot \sum_{i=2}^{d} E_{i, i-1}
$$

In other words, $X$ is the matrix

$$
\left(\begin{array}{ccccc}
0 & 0 & \ldots & 0 & 0 \\
t & 0 & \ldots & 0 & 0 \\
0 & t & \ldots & 0 & 0 \\
\vdots & \vdots & \ddots & \vdots & \vdots \\
0 & 0 & \ldots & t & 0
\end{array}\right)
$$

Since the first row of $X$ is zero, any matrix $M$ whose only nonzero entries are in its first column will satisfy $M X=0$, and is a potential candidate for $Y-X$. In particular, if we set $Y-X=t \cdot E_{1,1}$ or, equivalently

$$
Y=t \cdot E_{1,1}+X=t \cdot E_{1,1}+t \cdot \sum_{i=2}^{d} E_{i, i-1},
$$

then we have $(Y-X) X=t \cdot E_{1,1} X=0$. The matrices $X$ and $Y$ satisfy the conditions of Proposition 5.5, hence there is a unique homomorphism $\Phi_{d}: T(K) \rightarrow K[[t]]^{d \times d}$ sending $x=x(0,1)$ into $X$ and $y=x(-1,1)$ into $Y$. (Note that $\Phi_{1}: T(K) \rightarrow K[[t]]$ is precisely the map $\alpha$ introduced in Remark 5.4!)

Proposition 5.7 The restriction of $\Phi_{d}: T(K) \rightarrow K[[t]]^{d \times d}$ to the linear span of monomials $x^{i} y^{j}$ of degree at most $d-1$ is injective.

Proof: It is sufficient to show the same statement about products of the form $x^{i}(y-x)^{j}$ of degree at most $d-1$, since they span the same vector space and are equinumerous. We show this modified statement by explicitly calculating $X^{i}(Y-X)^{j}$ for $0 \leq i, j \leq d-1$. Concerning the powers of $X$ it is easy to show by induction that

$$
X^{i}=t^{i} \sum_{u=i+1}^{d} E_{u, u-i}
$$

holds for $1 \leq i \leq d-1$, while all higher powers of $X$ are zero. As a consequence

$$
X^{i}(Y-X)^{j}=t^{i} \cdot \sum_{u=i+1}^{d} E_{u, u-i} \cdot t^{j} \cdot E_{1,1}=t^{i+j} \cdot E_{i+1,1}
$$


for $1 \leq i \leq d-1$ and any positive $j$. Consider now any linear combination of the form

$$
M=\sum_{i=0}^{d-1} a_{i} \cdot X^{i}+\sum_{i=0}^{d-1} \sum_{j=1}^{d-1} b_{i, j} \cdot X^{i}(Y-X)^{j}
$$

with coefficients $a_{i}$ and $b_{i, j}$ from $K$, yielding $M=0$. By equations (13) and (14) we may write

$$
M=a_{0} \cdot I+\sum_{i=1}^{d-1} a_{i} \cdot t^{i} \sum_{u=i+1}^{d} E_{u, u-i}+\sum_{i=0}^{d-1} \sum_{j=1}^{d-1} b_{i, j} \cdot t^{i+j} \cdot E_{i+1,1},
$$

where $I$ is the identity matrix. For $1 \leq i \leq d-2$ we have $M_{d, d-i}=a_{i} \cdot t^{i}$ and so we must have $a_{1}=\cdots=a_{d-2}=0$. Since $M_{d, d}=a_{0}$, we also have $a_{0}=0$. Since $M_{d, 1}=a_{d-1} \cdot t^{d-1}+\sum_{j=1}^{d-1} b_{d-1, j} \cdot t^{d-1+j}$, we also have $a_{d-1}=b_{d-1,1}=b_{d-1,2}=\cdots=$ $b_{d-1, d-1}=0$. Finally, for $0 \leq i \leq d-2$ and $1 \leq j \leq d-1, b_{i, j}$ is the coefficient of $t^{i+j}$ in $M_{i+1,1}$ and must be zero.

Remark 5.8 It is worth noting that given $d_{1}<d_{2}$, the matrix $\Phi_{d_{1}}(x)$ may be obtained from $\Phi_{d_{2}}(x)$ by removing all entries except the ones in the first $d_{1}$ rows and columns. In other words, $\Phi_{d_{2}}$ is a lift of $\Phi_{d_{1}}$. Hence we may define $\Phi_{\infty}(x)$ as the infinite triangular matrix whose only nonzero entries are $\Phi_{\infty}(x)_{i, i-1}=t$ for $i=1,2, \ldots$. We may then set $\Phi_{\infty}(y)$ to be the matrix that is obtained from $\Phi_{\infty}(x)$ by changing the 0 in the first row, first column to $t$. The resulting infinite matrices $\Phi_{\infty}(x)$ and $\Phi_{\infty}(y)$ generate a subring of the ring of infinite lower triangular matrices over $K[[t]]$. We may also allow infinite linear combinations of products of the form $\Phi_{\infty}(x)^{i} \Phi_{\infty}(y)^{j}$ and perform the collection of terms entry by entry. The constant term of each entry of $\Phi_{\infty}(x)$ and $\Phi_{\infty}(y)$ being zero, for each entry the degree of $t$ dividing the entry increases as $i$ or $j$ increases. These infinite linear combinations provide a faithful representation of $T(K)$ as a subring of the ring of infinite lower triangular matrices over $K[[t]]$.

In order to apply Theorem 4.5 to our matrix representation, we now compute the products $X^{i} Y^{j}$. Obviously $Y^{j}=((Y-X)+X)^{j}$ may be written as the sum of all products consisting of altogether $j$ copies of $(Y-X)$ and $X$. Since $(Y-X) X=0$ and $X^{d}=0$, many of these products vanish, and we are left with

$$
Y^{j}=\sum_{u=0}^{\min (j, d-1)} X^{u}(Y-X)^{j-u}
$$

Using equations (13) and (14) we may rewrite this as

$$
Y^{j}=\sum_{u=0}^{\min (j-1, d-1)} t^{j} \cdot E_{u+1,1}+X^{j}
$$


Multiplying by $X^{i}$ from the left shifts all rows down by $i$, and multiplies all entries by $t^{i}$. Hence we obtain

$$
X^{i} Y^{j}=\sum_{u=i}^{\min (i+j-1, d-1)} t^{i+j} \cdot E_{u+1,1}+X^{i+j} .
$$

Here the first sum is empty if $j=0$ and the second power is zero if $j \geq d$. Moreover, for $i=0$, this formula yields exactly Eq. (15).

Theorem 5.9 Let $X$ and $Y$ be the matrices given by (11) and (12), respectively. Given a function $f \in R(\breve{T}(Q), K)$, the entries of the matrix

$$
\begin{aligned}
M & =\Phi_{d}(\phi(f))=\sum_{-1 \leq k<l<\infty} \frac{f(k, l)}{B(k, l)} X^{k+1} Y^{l-k-1} \text { are } \\
M_{p, q} & = \begin{cases}\frac{t^{p-q}}{B(p-q)} \cdot\left(\sum_{k=0}^{p-q-1}\left[\begin{array}{c}
p-q \\
k
\end{array}\right] f(k, p-q)+f(-1, p-q)\right) & \text { for } 2 \leq q \leq p, \\
\sum_{l=p-1}^{\infty} \frac{t^{l}}{B(l)} \cdot\left(f(-1, l)+\sum_{k=0}^{p-2}\left[\begin{array}{l}
l \\
k
\end{array}\right] f(k, l)\right) & \text { for } 1=q<p, \\
\sum_{l=0}^{\infty} \frac{f(-1, l) \cdot t^{l}}{B(l)} & \text { for } 1=q=p, \\
0 & \text { in all other cases. }\end{cases}
\end{aligned}
$$

Proof: Let us observe first that equation (16) may be rewritten as

$$
X^{k+1} Y^{l-k-1}=\sum_{u=k+1}^{\min (l-1, d-1)} t^{l} \cdot E_{u+1,1}+X^{l}
$$

where the first sum is empty if $l=k+1$ and the second power is zero if $l \geq d$. The formula is also correct if $k=-1$.

Assume first $q \geq 2$. None of the terms in the first sum of (17) contribute anything outside the first column, so these entries only consist of the contribution of the $X^{l}$ 's. By equation (13), $X^{l}$ contributes to $M_{p, q}$ exactly when $l=p-q$ (and, of course, we need $p-q \geq 0)$. Hence we obtain

$$
\begin{aligned}
M_{p, q} & =t^{p-q} \cdot \sum_{k=-1}^{p-q-1} \frac{f(k, p-q)}{B(k, p-q)} \\
& =t^{p-q} \cdot\left(\frac{f(-1, p-q)}{B(p-q)}+\sum_{k=0}^{p-q-1} \frac{f(k, p-q)}{B(k) B(p-q-k)}\right) \\
& =\frac{t^{p-q}}{B(p-q)} \cdot\left(f(-1, p-q)+\sum_{k=0}^{p-q-1}\left[\begin{array}{c}
p-q \\
k
\end{array}\right] \cdot f(k, p-q)\right),
\end{aligned}
$$

exactly as stated. 
Assume next $q=1$. Here we need to distinguish between the subcases $p=1$ and $p \geq 2$. The case $p=q=1$ is easier, since the top left entry in each $X^{k+1} Y^{l-k-1}$ satisfying $k \geq 0$ is zero by equations (17) and (13). Thus we only need to account for the contribution of $\sum_{l=0}^{\infty} \frac{f(-1, l)}{B(-1, l)} Y^{l}$, which gives

$$
M_{1,1}=\sum_{l=0}^{\infty} \frac{f(-1, l) \cdot t^{l}}{B(l)}
$$

Assume finally $q=1$ and $p \geq 2$. In this case both summands on the right hand side of equation (17) may contribute to $M_{p, 1}$. The term $X^{l}$ on the right hand side of Eq. (17) contributes to $M_{p, 1}$ exactly when $l=p-1$, and there is no restriction on the $k$ 's occurring, except for $k<l=p-1$. Thus the contribution of these terms to $M_{p, 1}$ is

$$
\sum_{k=-1}^{p-2} \frac{f(k, p-1)}{B(k, p-1)} \cdot t^{p-1}=\frac{t^{p-1}}{B(p-1)}\left(f(-1, l)+\sum_{k=0}^{p-2}\left[\begin{array}{c}
p-1 \\
k
\end{array}\right] f(k, l)\right) .
$$

The first sum on the right hand side of Eq. (17) contributes to $M_{p, q}$ exactly when $u=p-1$, and this may happen only if $k$ satisfies $k+1 \leq u=p-1$, i.e., $k \leq p-2$, and $l$ satisfies $l-1 \geq u=p-1$, i.e., $l \geq p$. (Note that when $k \leq p-2$ and $l \geq p$ then $k<l$ is automatically satisfied!) Hence the contribution of these terms to $M_{p, 1}$ is

$$
\begin{aligned}
\sum_{k=-1}^{p-2} \sum_{l=p}^{\infty} \frac{f(k, l)}{B(k, l)} t^{l} & =\sum_{l=p}^{\infty} \frac{f(-1, l)}{B(l)} t^{l}+\sum_{k=0}^{p-2} \sum_{l=p}^{\infty} \frac{f(k, l)}{B(k, l)} t^{l} \\
& =\sum_{l=p}^{\infty} \frac{f(-1, l)}{B(l)} t^{l}+\sum_{l=p}^{\infty} \frac{t^{l}}{B(l)} \sum_{k=0}^{p-2}\left[\begin{array}{l}
l \\
k
\end{array}\right] f(k, l) \\
& =\sum_{l=p}^{\infty} \frac{t^{l}}{B(l)}\left(f(-1, l)+\sum_{k=0}^{p-2}\left[\begin{array}{l}
l \\
k
\end{array}\right] f(k, l)\right)
\end{aligned}
$$

Adding up the two contributions yields exactly the stated formula for $M_{p, 1}$.

Remark 5.10 Since

$$
M_{1,1}=\sum_{l=0}^{\infty} \frac{f(-1, l) \cdot t^{l}}{B(l)}=\sum_{n \geq 0} \alpha_{R}(f)(n) \frac{t^{n}}{B(n)}
$$

is just the ordinary generating function of the restricted function $\alpha_{R}(f) \in R(Q, K)$, identities involving the matrices $M$ automatically specialize to identities in the classical setting. 


\section{Lower Eulerian binomial posets}

Recall that a poset $P$ is lower Eulerian if it has a unique minimum element $\hat{0}$ and for every $u \in P$ the interval $[\hat{0}, u]$ is Eulerian.

Using the notion of the augmented Tchebyshev operator we may rephrase Proposition 1.5 as follows:

Proposition 6.1 If $Q$ is a lower Eulerian poset, then so is $\check{T}(Q)$.

Assume now that $Q$ is a binomial and lower Eulerian poset. Then $\check{T}(Q)$ is also lower Eulerian. Since the rank of an interval of type $(k, l)$ in $\breve{T}(Q)$ is $l$, the value of the Möbius function on such an interval is $(-1)^{l}$. Using Theorem 4.5 and the fact that the Möbius function and the zeta function are inverses of each other, we obtain that the multiplicative inverse of

$$
\phi(\zeta)=\sum_{-1 \leq k<l<\infty} \frac{x(k, l)}{B(k, l)} \in T(K) \quad \text { is } \quad \phi(\mu)=\sum_{-1 \leq k<l<\infty}(-1)^{l} \cdot \frac{x(k, l)}{B(k, l)} \in T(K) .
$$

Substituting Definition 2.6 yields:

Corollary 6.2 If $Q$ is a lower Eulerian binomial poset, then the multiplicative inverse of

$$
\begin{aligned}
& \phi(\zeta)=\sum_{0 \leq l<\infty} \frac{x(-1, l)}{B(l)}+\sum_{0 \leq k<l<\infty} \frac{x(k, l)}{B(k) B(l-k)} \in T(K) \text { is } \\
& \phi(\mu)=\sum_{0 \leq l<\infty}(-1)^{l} \cdot \frac{x(-1, l)}{B(l)}+\sum_{0 \leq k<l<\infty}(-1)^{l} \cdot \frac{x(k, l)}{B(k) B(l-k)} \in T(K) .
\end{aligned}
$$

Using the representations $\Phi_{d}$ and Theorem 5.9, we obtain the following.

Corollary 6.3 If $Q$ is a lower Eulerian binomial poset, then the multiplicative inverse of the $d \times d$ matrix A given by

$$
A_{p, q}= \begin{cases}\frac{t^{p-q}}{B(p-q)} \cdot\left(\sum_{k=0}^{p-q-1}\left[\begin{array}{c}
p-q \\
k
\end{array}\right]+1\right) & \text { for } 2 \leq q \leq p, \\
\sum_{l=p-1}^{\infty} \frac{t^{l}}{B(l)} \cdot\left(1+\sum_{k=0}^{p-2}\left[\begin{array}{l}
l \\
k
\end{array}\right]\right) & \text { for } 1=q<p, \\
\sum_{l=0}^{\infty} \frac{t^{l}}{B(l)} & \text { for } 1=q=p, \\
0 & \text { in all other cases. }\end{cases}
$$

may be obtained by substituting $(-t)$ into $t$ in each entry of $A$. 
Example 6.4 Let the elements of $Q$ be all finite subsets of an infinite set, ordered by inclusion. This poset is locally finite, binomial, lower Eulerian, with factorial function $B(n)=n$ !. Corollary 6.2 yields that the multiplicative inverse of

$$
\begin{aligned}
& \sum_{0 \leq l<\infty} \frac{x(-1, l)}{l !}+\sum_{0 \leq k<l<\infty} \frac{x(k, l)}{k !(l-k) !} \in T(K) \text { is } \\
& \sum_{0 \leq l<\infty}(-1)^{l} \cdot \frac{x(-1, l)}{l !}+\sum_{0 \leq k<l<\infty}(-1)^{l} \cdot \frac{x(k, l)}{k !(l-k) !} \in T(K) .
\end{aligned}
$$

Let us rewrite the statement also in terms of Corollary 6.3. When $2 \leq q \leq p$, we obtain

$$
A_{p, q}=\frac{t^{p-q}}{(p-q) !} \cdot\left(\sum_{k=0}^{p-q-1}\left(\begin{array}{c}
p-q \\
k
\end{array}\right)+1\right)=\frac{(2 t)^{p-q}}{(p-q) !}
$$

For $1=q<p$ we obtain

$$
\begin{aligned}
& A_{p, 1}=\sum_{l=p-1}^{\infty} \frac{t^{l}}{l !} \cdot\left(1+\sum_{k=0}^{p-2}\left(\begin{array}{l}
l \\
k
\end{array}\right)\right) \\
& =\sum_{l=p-1}^{\infty} \frac{t^{l}}{l !}+\sum_{k=0}^{p-2} \frac{1}{k !} \sum_{l=p-1}^{\infty} \frac{t^{l}}{(l-k) !} \\
& =e^{t}-\sum_{l=0}^{p-2} \frac{t^{l}}{l !}+\sum_{k=0}^{p-2} \frac{t^{k}}{k !} \sum_{l=p-1}^{\infty} \frac{t^{l-k}}{(l-k) !} \\
& =e^{t}-\sum_{l=0}^{p-2} \frac{t^{l}}{l !}+\sum_{k=0}^{p-2} \frac{t^{k}}{k !}\left(\sum_{l=k}^{\infty} \frac{t^{l-k}}{(l-k) !}-\sum_{l=k}^{p-2} \frac{t^{l-k}}{(l-k) !}\right) \\
& =e^{t}-\sum_{l=0}^{p-2} \frac{t^{l}}{l !}+\sum_{k=0}^{p-2} \frac{t^{k}}{k !}\left(e^{t}-\sum_{m=0}^{p-2-k} \frac{t^{m}}{m !}\right) \\
& =e^{t}\left(1+\sum_{k=0}^{p-2} \frac{t^{k}}{k !}\right)-\sum_{k=0}^{p-2} \frac{t^{k}}{k !}-\sum_{k=0}^{p-2} \frac{t^{k}}{k !} \sum_{m=0}^{p-2-k} \frac{t^{m}}{m !} \\
& =e^{t}\left(1+\sum_{k=0}^{p-2} \frac{t^{k}}{k !}\right)-\sum_{k=0}^{p-2} \frac{t^{k}}{k !}-\sum_{m+k \leq p-2} \frac{t^{m+k}}{m ! k !} \\
& =e^{t}\left(1+\sum_{k=0}^{p-2} \frac{t^{k}}{k !}\right)-\sum_{k=0}^{p-2} \frac{t^{k}}{k !}-\sum_{l=0}^{p-2} \frac{t^{l}}{l !} \sum_{m=0}^{l}\left(\begin{array}{c}
l \\
m
\end{array}\right) \text {. }
\end{aligned}
$$


Using the binomial theorem, and collecting terms yields

$$
A_{p, 1}=e^{t}\left(1+\sum_{k=0}^{p-2} \frac{t^{k}}{k !}\right)-\sum_{k=0}^{p-2} \frac{\left(1+2^{k}\right) t^{k}}{k !}
$$

Finally, for $q=p=1$ we obtain

$$
A_{1,1}=\sum_{l=0}^{\infty} \frac{t^{l}}{l !}=e^{t}
$$

Corollary 6.5 The inverse of the $d \times d$ matrix given by

$$
A_{p, q}= \begin{cases}\frac{(2 t)^{p-q}}{(p-q) !} & \text { for } 2 \leq q \leq p, \\ e^{t}\left(1+\sum_{k=0}^{p-2} \frac{t^{k}}{k !}\right)-\sum_{k=0}^{p-2} \frac{\left(1+2^{k}\right) t^{k}}{k !} & \text { for } 1=q<p, \\ e^{t} & \text { for } 1=q=p, \\ 0 & \text { in all other cases. }\end{cases}
$$

may be obtained by substituting $(-t)$ into $t$ in each entry of $A$.

For $d=4$ this result yields that the inverse of the matrix

$$
\begin{aligned}
& \left(\begin{array}{cccc}
e^{t} & 0 & 0 & 0 \\
2 e^{t}-2 & 1 & 0 & 0 \\
(2+t) e^{t}-(2+3 t) & 2 t & 1 & 0 \\
\left(2+t+t^{2} / 2\right) e^{t}-\left(2+3 t+5 t^{2} / 2\right) & 2 t^{2} & 2 t & 1
\end{array}\right) \quad \text { is } \\
& \left(\begin{array}{cccc}
e^{-t} & 0 & 0 & 0 \\
2 e^{-t}-2 & 1 & 0 & 0 \\
(2-t) e^{-t}-(2-3 t) & -2 t & 1 & 0 \\
\left(2-t+t^{2} / 2\right) e^{-t}-\left(2-3 t+5 t^{2} / 2\right) & 2 t^{2} & -2 t & 1
\end{array}\right),
\end{aligned}
$$

which may be verified by hand.

Example 6.6 Consider the "ladder poset" $Q$ represented in figure 1. This is binomial, with factorial function

$$
B(n)= \begin{cases}2^{n-1} & \text { if } n \geq 1 \\ 1 & \text { if } n=0\end{cases}
$$


It is also lower Eulerian. (Intervals of $\check{T}(Q)$ for this particular $Q$ are the main subject of my paper [6].) The "binomial coefficients" $\left[\begin{array}{l}n \\ k\end{array}\right]$ are all equal to 2 , except for the two extreme cases $\left[\begin{array}{l}n \\ 0\end{array}\right]=\left[\begin{array}{l}n \\ n\end{array}\right]=1$. By Corollary 6.2 we obtain that the multiplicative inverse of

$$
\begin{aligned}
& \sum_{0 \leq l<\infty} \frac{x(-1, l)}{2^{l-1}}+\sum_{0 \leq k<l<\infty} \frac{x(k, l)}{2^{l-2}} \in T(K) \text { is } \\
& \sum_{0 \leq l<\infty}(-1)^{l} \cdot \frac{x(-1, l)}{2^{l-1}}+\sum_{0 \leq k<l<\infty}(-1)^{l} \cdot \frac{x(k, l)}{2^{l-2}} \in T(K) .
\end{aligned}
$$

Let us rewrite the statement also in terms of Corollary 6.3. For $2 \leq q<p$, we obtain

$$
\begin{aligned}
A_{p, q} & =\frac{t^{p-q}}{2^{p-q-1}} \cdot\left(\sum_{k=0}^{p-q-1}\left[\begin{array}{c}
p-q \\
k
\end{array}\right]+1\right)=\frac{t^{p-q}}{2^{p-q-1}} \cdot 2 \cdot(p-q) \\
& =4 \cdot(p-q) \cdot\left(\frac{t}{2}\right)^{p-q},
\end{aligned}
$$

while $A_{q, q}=1$ for $q \geq 2$. Assume next $1=q<p$. Then

$$
\begin{aligned}
A_{p, 1} & =\sum_{l=p-1}^{\infty} \frac{t^{l}}{2^{l-1}} \cdot\left(1+\sum_{k=0}^{p-2}\left[\begin{array}{l}
l \\
k
\end{array}\right]\right)=\sum_{l=p-1}^{\infty} \frac{t^{l}}{2^{l-1}} \cdot 2 \cdot(p-1) \\
& =4 \cdot(p-1) \cdot \sum_{l=p-1}^{\infty} \frac{t^{l}}{2^{l}}=(p-1) \cdot\left(\frac{t}{2}\right)^{p-1} \cdot \frac{8}{2-t} .
\end{aligned}
$$

Finally for $1=q=p$ we obtain

$$
A_{1,1}=1+\sum_{l=1}^{\infty} \frac{t^{l}}{2^{l-1}}=\frac{2+t}{2-t} .
$$

Corollary 6.7 The inverse of the $d \times d$ matrix given by

$$
A_{p, q}= \begin{cases}1 & \text { for } 2 \leq q=p, \\ 4 \cdot(p-q) \cdot\left(\frac{t}{2}\right)^{p-q} & \text { for } 2 \leq q<p, \\ (p-1) \cdot\left(\frac{t}{2}\right)^{p-1} \cdot \frac{8}{2-t} & \text { for } 1=q<p, \\ \frac{2+t}{2-t} & \text { for } 1=q=p, \\ 0 & \text { in all other cases. }\end{cases}
$$

may be obtained by substituting $(-t)$ into $t$ in each entry of $A$. 
For $d=4$ this yields

$$
\left(\begin{array}{cccc}
(2+t) /(2-t) & 0 & 0 & 0 \\
4 t /(2-t) & 1 & 0 & 0 \\
4 t^{2} /(2-t) & 2 t & 1 & 0 \\
3 t^{3} /(2-t) & 2 t^{2} & 2 t & 1
\end{array}\right)^{-1}=\left(\begin{array}{cccc}
(2-t) /(2+t) & 0 & 0 & 0 \\
-4 t /(2+t) & 1 & 0 & 0 \\
4 t^{2} /(2+t) & -2 t & 1 & 0 \\
-3 t^{3} /(2+t) & 2 t^{2} & -2 t & 1
\end{array}\right)
$$

\section{The Möbius function of $\check{T}(\mathbb{N})$}

As a final illustration of the power of Theorem 5.9, we calculate the inverse of a lifted sequence of $d \times d$ matrices using the fact that the Möbius function of $\check{T}(\mathbb{N})$ is the multiplicative inverse of its zeta function. It is very easy to show that the Möbius function $\mu$ of $\check{T}(\mathbb{N})$ satisfies

$$
\mu(k, l)= \begin{cases}1 & \text { if }(k, l) \in\{(-1,0),(1,2)\} \\ -1 & \text { if } l=1, \\ 0 & \text { for all other values of }(k, l) .\end{cases}
$$

The reader familiar with Möbius function computations may use figure 2 to fill in the picture and notice that, except for four elements at the bottom, all others need to be labeled with 0 .

The binomial poset $\mathbb{N}$ has factorial function $B(l)=1$ and "binomial coefficients" $\left[\begin{array}{l}n \\ k\end{array}\right]=1$. Let $A$ be the $d \times d$ matrix associated to the zeta function of $\breve{T}(\mathbb{N})$ under $\Phi_{d}$. For $2 \leq q \leq p$ Theorem 5.9 yields

$$
A_{p, q}=t^{p-q} \cdot\left(\sum_{k=0}^{p-q-1}\left[\begin{array}{c}
p-q \\
k
\end{array}\right]+1\right)=(p-q+1) \cdot t^{p-q}
$$

For $1=q<p$, Theorem 5.9 yields

$$
A_{p, 1}=\sum_{l=p-1}^{\infty} t^{l} \cdot\left(1+\sum_{k=0}^{p-2}\left[\begin{array}{l}
l \\
k
\end{array}\right]\right)=p \cdot \sum_{l=p-1}^{\infty} t^{l}=\frac{p \cdot t^{p-1}}{1-t}
$$

Finally, for $1=q=p$ we have

$$
A_{1,1}=\sum_{l=0}^{\infty} t^{l}=\frac{1}{1-t}
$$


The inverse of $A$ is the image of the Möbius function under $\Phi_{d}$. Using Eq. (18) and Theorem 5.9 we obtain:

$$
A_{p, q}^{-1}= \begin{cases}t^{2} & \text { for } 1 \leq q<p \text { and } p-q=2 \\ -2 t & \text { for } 1 \leq q<p \text { and } p-q=1 \\ 1 & \text { for } 2 \leq q=p \\ 1-t & \text { for } 1=q=p \\ 0 & \text { in all other cases. }\end{cases}
$$

For $d=5$ we obtain

$$
\left(\begin{array}{ccccc}
1 /(1-t) & 0 & 0 & 0 & 0 \\
2 t /(1-t) & 1 & 0 & 0 & 0 \\
3 t^{2} /(1-t) & 2 t & 1 & 0 & 0 \\
4 t^{3} /(1-t) & 3 t^{2} & 2 t & 1 & 0 \\
5 t^{4} /(1-t) & 4 t^{3} & 3 t^{2} & 2 t & 1
\end{array}\right)^{-1}=\left(\begin{array}{ccccc}
1-t & 0 & 0 & 0 & 0 \\
-2 t & 1 & 0 & 0 & 0 \\
t^{2} & -2 t & 1 & 0 & 0 \\
0 & t^{2} & -2 t & 1 & 0 \\
0 & 0 & t^{2} & -2 t & 1
\end{array}\right)
$$

As a consequence of the Möbius function vanishing on all but finitely many elements, $A^{-1}$ is "almost diagonal": only those entries are nonzero for which the row number exceeds the column number by at least 0 and and at most 2 .

\section{8. "One-dimensional" representations of the Tchebyshev algebra}

It is relatively easy to essentially describe all "one-dimensional" representations of $T(K)$, that is, all homomorphisms $\psi: T(K) \rightarrow K[[t]]$. Given such a homomorphism $\psi$, the formal power series $\psi(x)$ and $\psi(y)$ must satisfy

$$
\psi(y) \cdot \psi(x)=\psi(x)^{2}
$$

(Here we identify $T(K)$ with $K\langle\langle x, y\rangle\rangle / \mathbf{c l}\left(y x-x^{2}\right)$.) Since $K[[t]]$ is an integral domain, we must have either $\psi(x)=0$ or $\psi(y)=\psi(x)$. In the first case, the kernel of $\psi$ contains the ideal generated by $x$, and the homomorphism factors through $T(K) /(x) \cong K[[y]]$. (Although $T(K)$ is not commutative, each of its elements may be written as an infinite linear combination of terms $x^{i} y^{j}$, and any such expression containing only terms $x^{i} y^{j}$ with a positive $i$ is a multiple of $x$. Hence there is no need to "close" the ideal generated by $x$.) The ring $K[[y]]$ is obviously isomorphic to $K[[t]]$ so we may use the factoring of $\psi$ through $K[[y]]$ to obtain a description $\psi_{1} \circ \alpha$, where $\psi_{1}$ is an endomorphism of $K[[t]]$, and $\alpha$ is the "canonical homomorphism" $\alpha: T(K) \rightarrow K[[t]]$ introduced in Remark 5.4, sending $x$ into 0 and $y$ into $t$. 
Consider now the second case, when $\psi(x)=\psi(y)$. In this case $\psi$ factors through $T(K) /(x-y)$.

Lemma 8.1 The factor of $T(K) \cong K\langle\langle x, y\rangle\rangle / \operatorname{cl}\left(y x-x^{2}\right)$ by the ideal generated by $y-x$ is isomorphic to $K[[x]]$.

Proof: Using $y x=x^{2}$, it is easy to show by induction that $y^{i-1} x=x^{i}$ holds for $i \geq 2$ in $T(K)$. Hence we have

$$
y^{j}-x^{j}=y^{j-1}(y-x) \text { for } j \geq 1 .
$$

Therefore any infinite linear combination $\sum_{i, j \geq 0} a_{i, j} x^{i} y^{j}$ is congruent modulo the ideal generated by $y-x$ to $\sum_{n=0}^{\infty} \sum_{i=0}^{n} a_{i, n-i} x^{n}$, since their difference

$$
\begin{aligned}
\sum_{i, j \geq 0} a_{i, j} x^{i} y^{j}-\sum_{n=0}^{\infty} \sum_{i=0}^{n} a_{i, n-i} x^{n} & =\sum_{n=0}^{\infty} \sum_{i=0}^{n-1} a_{i, n-i} x^{i}\left(y^{n-i}-x^{n-i}\right) \\
& =\sum_{n=0}^{\infty} \sum_{i=0}^{n-1} a_{i, n-i} x^{i} y^{n-i-1}(y-x) \\
& =\sum_{i=0}^{\infty} \sum_{j=1}^{\infty} a_{i, j} x^{i} y^{j-1} \cdot(y-x)
\end{aligned}
$$

is an element of the ideal generated by $y-x$.

Again, the ring $K[[x]]$ is isomorphic to $K[[t]]$ and so $\psi$ is of the form $\psi_{1} \circ \beta$ where $\psi_{1}$ is an endomorphism of $K[[t]]$ and $\beta$ is the homomorphism $\beta: T(K) \rightarrow K[[t]]$ sending both $x$ and $y$ into $t$. To summarize, we have obtained the following theorem.

Theorem 8.2 Every homomorphism $\psi: T(K) \rightarrow K[[t]]$ is of the form $\psi_{1} \circ \psi_{2}$, where $\psi_{1}$ is an endomorphism of $K[[t]]$, and $\psi_{2}$ is either the homomorphism $\alpha: T(K) \rightarrow K[[t]]$ sending $x$ into 0 and $y$ into $t$, or the homomorphism $\beta: T(K) \rightarrow K[[t]]$ sending both $x$ and $y$ into $t$.

A good reference on the endomorphisms of a commutative formal power series ring is Chapter 3 of Brewer's book [2]. We now take a closer look at the homomorphisms $\alpha$ and $\beta$. Given a binomial poset $Q$ and a function $f \in R(\check{T}(Q), K)$, we have

$$
\alpha\left(\sum_{-1 \leq k<l<\infty} \frac{f(k, l) x^{k+1} y^{l-k-1}}{B(k, l)}\right)=\sum_{l=0}^{\infty} \frac{f(-1, l) t^{l}}{B(l)}
$$


thus $\alpha$ induces a homomorphism. Finally, consider the effect of $\beta$. Given a binomial poset $Q$ and a function $f \in R(\breve{T}(Q), K)$, we have

$$
\begin{aligned}
\beta\left(\sum_{-1 \leq k<l<\infty} \frac{f(k, l) x^{k+1} y^{l-k-1}}{B(k, l)}\right) & =\sum_{l=0}^{\infty} t^{l} \cdot \sum_{k=-1}^{l-1} \frac{f(k, l)}{B(k, l)} \\
& =\sum_{l=0}^{\infty} \frac{t^{l}}{B(l)} \cdot\left(f(-1, l)+\sum_{k=0}^{l-1}\left[\begin{array}{l}
l \\
k
\end{array}\right] f(k, l)\right)
\end{aligned}
$$

Therefore $\beta$ induces a homomorphism $\beta_{R}: R(\check{T}(Q), K) \rightarrow R(Q, K)$ given by

$$
\beta_{R}(f)(l)=f(-1, l)+\sum_{k=0}^{l-1}\left[\begin{array}{l}
l \\
k
\end{array}\right] f(k, l) .
$$

One would be tempted to look for a homomorphism $\beta_{I}: I(\check{T}(Q), K) \rightarrow I(Q, K)$ that extends the definition of $\beta_{R}$, in analogy to (8). Unfortunately, using the most plausible extension, one seems to run into a difficulty with preserving multiplication. We are still able to extend $\beta$ to the standard algebra of a poset.

Definition 8.3 Let $P$ be a locally finite partially ordered set, and $K$ a field. We define the standard algebra $S(P, K)$ of $P$ as the set of those $f \in I(P, K)$ that assign the same value to isomorphic intervals.

According to Proposition 4.13 in Aigner's book [1, Chapter IV, Section 1] the standard algebra is a subalgebra of the incidence algebra. Moreover, if an element of the standard algebra is invertible in the incidence algebra, then it is already invertible in the standard algebra. It is also evident that $R(P, K)$ is a subalgebra of $S(P, K)$.

Lemma 8.4 Given any locally finite partially ordered set $Q$ with minimum element $\hat{0}$, the map $\beta_{S}: S(\check{T}(Q), K) \rightarrow S(Q, K)$ given by

$$
\beta_{S}(f)([x, y])=\sum_{z \in\{-1\} \cup[x, y)} f([(\widehat{-1}, x),(z, y)]) .
$$

is a homomorphism. Furthermore, if $Q$ is a binomial poset, then restriction of $\beta_{S}$ to $R(\breve{T}(Q), K)$ is $\beta_{R}$.

Proof: It is easy to verify that $\beta_{S}$ is compatible with addition, and that, for a binomial poset $Q$, its restriction to $R(\breve{T}(Q), K)$ is $\beta_{R}$. We only need to show that $\beta_{S}$ is compatible with the convolution. Given $(x, y) \in \check{T}(Q)$ we have $x<y$ and

$$
\begin{aligned}
\beta_{S}(f \cdot g)([x, y]) & =\sum_{z \in\{\{-1\} \cup[x, y)}(f \cdot g)([(\widehat{-1}, x),(z, y)]) \\
& =\sum_{z \in\{-1\}\} \cup[x, y)} \sum_{(\widehat{-1}, x) \leq(u, v) \leq(z, y)} f([(\widehat{-1}, x),(u, v)]) g([(u, v),(z, y)])
\end{aligned}
$$




$$
\begin{aligned}
= & \sum_{x \leq v \leq y} \sum_{u \in\{\widehat{-1}\} \cup[x, v)} f([(\widehat{-1}, x),(u, v)]) \\
& \times\left(g([(u, v),(u, y)])+\sum_{v \leq z<y} g([(u, v),(z, y)])\right) .
\end{aligned}
$$

Given any fixed $u \in\{\widehat{-1}\} \cup[x, v)$, the interval $[(u, v),(u, y)]$ is isomorphic to $[(\widehat{-1}, v)$, $(\widehat{-1}, y)]$ (and to the interval $[v, y]$ of $Q$, by Lemma 1.4). Given also a $z \in[v, y)$, the interval $[(u, v),(z, y)]$ is isomorphic to the interval $[(\widehat{-1}, v),(z, y)]$ by Lemma 1.7. Since $g$ belongs to the standard algebra, we obtain

$$
g([(u, v),(u, y)])+\sum_{v \leq z<y} g([(u, v),(z, y)])=\sum_{z \in\{\widehat{-1}\} \cup[v, y)} g([(\widehat{-1}, v),(z, y)]) .
$$

Thus

$$
\begin{aligned}
\beta_{S}(f \cdot g)([x, y])= & \sum_{x \leq v \leq y} \sum_{u \in\{\widehat{-1}\} \cup[x, v)} f([(\widehat{-1}, x),(u, v)]) \\
& \times\left(\sum_{z \in\{\widehat{-1}\} \cup[v, y)} g([(\widehat{-1}, v),(z, y)])\right) . \\
= & \sum_{x \leq v \leq y} \beta_{S}(f)([x, v]) \cdot \beta_{S}(g)([v, y])
\end{aligned}
$$

which is exactly the definition of $\left(\beta_{S}(f) \cdot \beta_{S}(g)\right)([x, y])$.

The combinatorial meaning of Theorem 8.2 is that for a binomial poset $Q, \alpha_{I}$ and $\beta_{S}$ are the only "essentially interesting" homomorphisms between the incidence algebras or standard algebras of $\breve{T}(Q)$ and $Q$ : at the level of generating functions, any other homomorphism would be either meaningless, or induce the same formulas, modulo the endomorphisms of $K[[t]]$.

Remark 8.5 We have seen that the matrix representations $\Phi_{d}$ lift the map $\alpha$ and they "converge" to a faithful representation of the Tchebyshev algebra. It would be interesting to find out whether a similar sequence of matrix representations could be constructed to lift $\beta$.

\section{Tchebyshev polynomials and derivative polynomials}

As noted in Proposition 1.9, the Tchebyshev polynomial $T_{n}(x)$ of the first kind is connected to the face numbers of the order complex of any open interval of type $(n, n+1)$ in the augmented Tchebyshev transform of the "ladder poset" represented in figure 1 . We may use this proposition to associate a sequence of generalized Tchebyshev polynomials to each binomial poset $Q$. 
Definition 9.1 Given a binomial poset $Q$, we define the $n$-th $Q$-generalized Tchebyshev polynomial $T_{n}^{Q}(s)$ by setting

$$
T_{n}^{Q}(s)=\sum_{j=0}^{n} f_{j-1}(\triangle(((\widehat{-1}, \hat{0}),(u, v)))) \cdot\left(\frac{s-1}{2}\right)^{j}
$$

where $(u, v) \in \check{T}(Q)$ is any element of type $(n, n+1)$.

Remark 9.2 Definition 9.1 may be rephrased in terms of the Tchebyshev algebra as follows. The number of $(j-1)$-dimensional faces in an order complex is the same as the number of $j$-element chains, which may be expressed using the zeta function, as noted by Stanley in [15, Example 3.15.9]. It is easy to verify that $T_{n}^{Q}(s)$ is the coefficient of $x^{n+1}=x(n, n+1)$ in

$$
\phi\left(\sum_{n=0}^{\infty}(\zeta-1)^{n+1} \cdot\left(\frac{s-1}{2}\right)^{n}\right)
$$

where $\phi: R(\check{T}(Q), K) \rightarrow T(K)$ is the algebra isomorphism from Theorem 4.5. Note that sending all monomials containing a factor of $y$ to zero is not a homomorphism, so at best one could use some sort of "umbral calculus" here!

Definition 9.1 is especially appealing since, according to Definition 2.5, if $(u, v)$ has type $(n, n+1)$ then for any $\left(u^{\prime}, v^{\prime}\right) \in((-1,0),(u, v))$, the type of the interval $\left(\left(u^{\prime}, v^{\prime}\right),(u, v)\right)$ is also of the form $\left(n^{\prime}, n^{\prime}+1\right)$ for some $n^{\prime}$. More precisely, we have the recursion formula

$$
T_{n}^{Q}(s)=1+\frac{s-1}{2} \cdot \sum_{m=1}^{n}\left[\begin{array}{l}
n \\
m
\end{array}\right] \sum_{k=0}^{m}\left[\begin{array}{l}
m \\
k
\end{array}\right] T_{n-m}^{Q}(s) .
$$

In fact, calculating $T_{n}^{Q}(s)$ involves summing over all partial chains in an open interval of type $(n, n+1)$ in such a way that each element in each partial chain contributes a factor of $(s-1) / 2$. The summand 1 on the right hand side of (19) corresponds to choosing the empty chain, while the sum over $m$ corresponds to choosing the least element $(u, v)$ of a partial chain such that $\rho(v)=m$. There are $\left[\begin{array}{c}n \\ m\end{array}\right]$ ways to choose $v$, and the second sum corresponds to choosing an appropriate $u$ for a fixed $v$, satisfying $\rho(u)=k$. (Note that $u=v$ is not allowed, yet we include the term $\left[\begin{array}{c}m \\ m\end{array}\right]=1$ to account for the case $\rho(u)=-1$.) Finally the term $T_{n-m}^{Q}(s)$ corresponds to choosing the rest of the chain above $(u, v)$, in an interval of type $(n-m, n+1-m)$.

Equation (19) may be used to compute the "exponential generating function" of the polynomials $T_{n}^{Q}(s)$. In fact, adding $(s-1) / 2 \cdot T_{n}^{Q}(s)$, multiplying both sides by $t^{n} / B(n)$, and summing over $n$ from 0 to infinity yields:

$$
\frac{s+1}{2} \sum_{n=0}^{\infty} T_{n}^{Q}(s) \frac{t^{n}}{B(n)}=\sum_{n=0}^{\infty} \frac{t^{n}}{B(n)}+\frac{s-1}{2} \sum_{n=0}^{\infty} T_{n}^{Q}(s) \frac{t^{n}}{B(n)} \cdot \sum_{m=0}^{\infty} \sum_{k=0}^{m}\left[\begin{array}{l}
m \\
k
\end{array}\right] \frac{t^{m}}{B(m)} .
$$


We may solve this for $\sum_{n=0}^{\infty} T_{n}^{Q}(s)\left(t^{n} / B(n)\right)$ and get

$$
\sum_{n=0}^{\infty} T_{n}^{Q}(s) \frac{t^{n}}{B(n)}=\frac{\sum_{n=0}^{\infty} \frac{t^{n}}{B(n)}}{\frac{s+1}{2}-\frac{s-1}{2} \sum_{m=0}^{\infty} \sum_{k=0}^{m}\left[\begin{array}{c}
m \\
k
\end{array}\right] \frac{t^{m}}{B(m)}}
$$

If $Q$ is the "ladder" poset in figure 1 then (20) may be simplified to

$$
1+\sum_{n=1}^{\infty} T_{n}(s) \frac{t^{n}}{2^{n-1}}=\frac{4-t^{2}}{4-4 s t+t^{2}}
$$

a well-known formula. If $Q$ is the partially ordered set of all finite subsets of an infinite set, ordered by inclusion, then (20) takes the form

$$
\sum_{n=0}^{\infty} T_{n}^{Q}(s) \frac{t^{n}}{n !}=\frac{e^{t}}{\frac{s+1}{2}-\frac{s-1}{2} e^{2 t}}=\frac{1}{\frac{s+1}{2} e^{-t}-\frac{s-1}{2} e^{t}} .
$$

Replacing $t$ with it (where $i=\sqrt{-1}$ ) yields

$$
\sum_{n=0}^{\infty} T_{n}^{Q}(s) \frac{(i t)^{n}}{n !}=\frac{1}{\cos (t)-s \cdot i \cdot \sin (t)} .
$$

The function $1 /(\cos (t)-u \sin (t))$ may be written as

$$
\frac{1}{\cos (t)-u \cdot \sin (t)}=\sum_{n=0}^{\infty} Q_{n}(u) \frac{t^{n}}{n !}
$$

where the derivative polynomials $Q_{n}(u)$ are defined by

$$
\frac{d^{n}}{d u^{n}} \sec (u)=Q_{n}(\tan u) \sec u
$$

as stated in the introduction of Hoffman's paper [9]. ${ }^{2}$

Corollary 9.3 For the partially ordered set $Q$ consisting of the finite subsets of an infinite set, we have

$$
T_{n}^{Q}(s)=i^{-n} Q_{n}(s \cdot i)
$$

where the polynomials $Q_{n}(u)$ are the derivative polynomials given in (21).

This example shows that the sequence $T_{n}^{Q}(s)$ does not have to be a sequence of orthogonal polynomials in general, but is likely to have many interesting combinatorial aspects, as witnessed by Hoffman's paper [9]. The interested reader may find a different generalization 
of the methods of [6] in [7] which yields orthogonal polynomial sequence generalizations of the poset constructions associated to Tchebyshev polynomials.

\section{Appendix A. The proof of Theorem 1.10}

Part $(i)$ of Theorem 1.10 is a trivial consequence of Lemma 1.4. To show ( $i i)$, observe first that we may assume that $y_{2}$ covers $x_{2}$. In the contrary event, any $(u, v) \in\left(\left(x_{1}, y_{2}\right),\left(x_{2}, y_{2}\right)\right)$ either satisfies $v \leq x_{2}$ or $u=x_{2}$. Let $\dot{x}_{2}$ be any element covering $x_{2}$. If $v \leq x_{2}$ then $(u, v)$ belongs to $\left(\left(x_{1}, y_{2}\right),\left(x_{2}, \dot{x}_{2}\right)\right)$, and it is easy to see that this open interval contains the same elements, no matter which $\dot{x}_{2}$ we choose. Moreover, any $(u, v)$ satisfying $v \leq x_{2}$ is less than any $\left(u^{\prime}, v^{\prime}\right)$ satisfying $u^{\prime}=x_{2}$. In analogy to Lemma 1.4 , the set of $(u, v)$ 's satisfying $u=x_{2}$ and $(u, v)<\left(x_{2}, y_{2}\right)$ is easily seen to be isomorphic to the open interval $\left(x_{2}, y_{2}\right) \subset Q$. Hence the order complex of $\left(\left(x_{1}, y_{1}\right),\left(x_{2}, y_{2}\right)\right)$ is isomorphic to the join of $\triangle\left(\left(\left(x_{1}, y_{1}\right),\left(x_{2}, \dot{x}_{2}\right)\right)\right)$ and of $\triangle\left(\left(x_{2}, y_{2}\right)\right)$. Since the suspension operation "commutes" with taking the join, i.e., $\Sigma\left(\triangle_{1}\right) * \triangle_{2}=\Sigma\left(\triangle_{1} * \triangle_{2}\right)=\triangle_{1} * \Sigma\left(\triangle_{2}\right)$ holds for any pair of simplicial complexes on disjoint vertex sets, since for $x_{2} \prec \dot{x}_{2}$ we have $\triangle\left(\left(x_{2}, \dot{x}_{2}\right)\right)=\{\emptyset\}$, and since $\triangle *\{\emptyset\}=\triangle$ holds for any simplicial complex $\Delta$, we are done if we are able to show our statement for the case $x_{2} \prec y_{2}$. In that case (ii) is equivalent to stating that $\Delta\left(\left(\left(x_{1}, y_{1}\right),\left(x_{2}, y_{2}\right)\right)\right)$ is isomorphic to a triangulation of $\Sigma\left(\triangle\left(\left(y_{1}, x_{2}\right)\right)\right)$. We show this by proving the following generalization of [6, Proposition 4.3].

Lemma A.1 Assume $x_{1}<y_{1} \leq x_{2} \prec y_{2}$, and consider the Euclidean space $E$ whose orthonormal basis is the set $\left\{x_{1}\right\} \cup\left[y_{1}, x_{2}\right)$. Realize each face of $\triangle\left(\left\{x_{1}\right\} \cup\left[y_{1}, x_{2}\right)\right)$ in $E$ as the convex hull of its vertices. Let $\eta:\left(\left(x_{1}, y_{1}\right),\left(x_{2}, y_{2}\right)\right) \rightarrow E$ be given by

$$
\eta(u, v)= \begin{cases}u & \text { if } v=x_{2} \\ (u+v) / 2 & \text { if } v<x_{2}\end{cases}
$$

Then $\eta$ induces a geometric realization of $\triangle\left(\left[\left(x_{1}, y_{1}\right),\left(x_{2}, y_{2}\right)\right)\right)$ as a triangulation of the realization of $\triangle\left(\left\{x_{1}\right\} \cup\left[y_{1}, x_{2}\right)\right)$.

Proof: Since $\left\{x_{1}\right\} \cup\left[y_{1}, x_{2}\right)$ is the basis of the space, the faces of $\Delta\left(\left\{x_{1}\right\} \cup\left[y_{1}, x_{2}\right)\right)$ are represented as convex hulls of affine independent vertex sets. The description given in the first part of Lemma 2.3 may be generalized to arbitrary chains in $\left[\left(x_{1}, y_{1}\right),\left(x_{2}, y_{2}\right)\right)$ as follows: given $\sigma=\left\{\left(u_{1}, v_{1}\right), \ldots,\left(u_{k}, v_{k}\right)\right\} \subseteq\left[\left(x_{1}, y_{1}\right),\left(x_{2}, y_{2}\right)\right)$ satisfying $\left(u_{0}, v_{0}\right):=$ $\left(x_{1}, y_{1}\right) \leq\left(u_{1}, v_{1}\right)<\cdots<\left(u_{k}, v_{k}\right)<\left(x_{2}, y_{2}\right)$, we must have $y_{1} \leq v_{0}<v_{1}<\cdots<v_{k} \leq$ $x_{2}$ (here we use $x_{2} \prec y_{2}$ ) and, for each $i \geq 1$, either $u_{i}=u_{i-1}$ or $v_{i-1} \leq u_{i}<v_{i}$ must hold. Using this observation it is easy to show that the set $\left\{u_{1}, v_{1}, \ldots, u_{k}, v_{k}\right\}$ must be a partial chain in $\left\{x_{1}\right\} \cup\left[y_{1}, x_{2}\right)$, and so for any $\sigma \in \Delta\left(\left[\left(x_{1}, y_{1}\right),\left(x_{2}, y_{2}\right)\right)\right)$, the convex hull of $\eta(\sigma)$ is contained in the geometric realization of some face of $\triangle\left(\left\{x_{1}\right\} \cup\left[y_{1}, x_{2}\right)\right)$. Next we show that each $\sigma \in \Delta\left(\left[\left(x_{1}, y_{1}\right),\left(x_{2}, y_{2}\right)\right)\right)$ is realized with affine independent vertices. 
If not, consider a smallest counterexample $\sigma=\left\{\left(u_{1}, v_{1}\right), \ldots,\left(u_{k}, v_{k}\right)\right\}$ satisfying

$$
\sum_{i=1}^{k} \alpha_{i} \cdot \eta\left(u_{i}, v_{i}\right)=0 \quad \text { and } \quad \sum_{i=1}^{k} \alpha_{i}=1
$$

By the minimality of $\sigma$ we may assume that all $\alpha_{i}$ 's are nonzero. By the nature of the map $\eta$, if any $z \in\left\{x_{1}\right\} \cup\left[x_{2}, y_{2}\right)$ appears in the list $\left(u_{1}, v_{1}, \ldots, u_{k}, v_{k}\right)$ only once then we reach a contradiction, since then the $z$-component of $\sum_{i=1}^{k} \alpha_{i} \cdot \eta\left(u_{i}, v_{i}\right)$ is nonzero. In particular, $u_{1}$ must occur for a second time in the list, and so we must have $k \geq 2$ and $u_{2}=u_{1}$. But then $v_{1}$ satisfies $u_{1}=u_{2}<v_{1}<v_{2}$, it is different from all other $u_{i}$ 's and $v_{j}$ 's, and it is less than $x_{2}$. This contradiction proves that $\eta$ induces a faithful geometric realization of $\triangle\left(\left[\left(x_{1}, y_{1}\right),\left(x_{2}, y_{2}\right)\right)\right)$.

We are left to show that the convex hull of every facet $F$ of $\triangle\left(\left\{x_{1}\right\} \cup\left[y_{1}, x_{2}\right)\right)$ is a union of convex hulls of sets $\eta(\sigma)$ for some faces $\sigma \in \Delta\left(\left[\left(x_{1}, y_{1}\right),\left(x_{2}, y_{2}\right)\right)\right)$. We show this by induction on the number of elements in $\left\{x_{1}\right\} \cup\left[x_{2}, y_{2}\right)$. A facet $F$ of $\triangle\left(\left\{x_{1}\right\} \cup\left[y_{1}, x_{2}\right)\right)$ is of the form $x_{1} \prec y_{1} \prec \dot{y}_{1} \prec \ldots$, where $\dot{y}_{1}$ covers $y_{1}$ even in the original poset $Q$ from which we take our intervals. By our induction hypothesis $\eta$ induces a geometric realization of $\triangle\left(\left[\left(x_{1}, \dot{y}_{1}\right),\left(x_{2}, y_{2}\right)\right)\right)$ as a triangulation of $\triangle\left(\left\{x_{1}\right\} \cup\left[\dot{y}_{1}, x_{2}\right)\right)$ and a geometric realization of $\Delta\left(\left[\left(y_{1}, \dot{y}_{1}\right),\left(x_{2}, y_{2}\right)\right)\right)$ as a triangulation of $\Delta\left(\left\{y_{1}\right\} \cup\left[\dot{y}_{1}, x_{2}\right)\right)$. (Note that both $\triangle\left(\left[\left(x_{1}, \dot{y}_{1}\right),\left(x_{2}, y_{2}\right)\right)\right)$ and $\triangle\left(\left[\left(y_{1}, \dot{y}_{1}\right),\left(x_{2}, y_{2}\right)\right)\right)$ are subcomplexes of $\triangle\left(\left[\left(x_{1}, y_{1}\right),\left(x_{2}, y_{2}\right)\right)\right)$, the sets $\left\{x_{1}\right\} \cup\left[\dot{y}_{1}, x_{2}\right)$ and $\left\{y_{1}\right\} \cup\left[\dot{y}_{1}, x_{2}\right)$ are subsets of $\left\{x_{1}\right\} \cup\left[y_{1}, x_{2}\right)$, spanning Euclidean subspaces of $E$, and the function $\eta$ is defined by the same rule in all three situations, since the role of $x_{2}$ is the same each time.) Hence the convex hull of $F \backslash\left\{x_{1}\right\}$ and the convex hull of $F \backslash\left\{y_{1}\right\}$ are unions of convex hulls of sets $\eta(\sigma)$ where each $\sigma$ belongs to (a subcomplex of $) \triangle\left(\left[\left(x_{1}, y_{1}\right),\left(x_{2}, y_{2}\right)\right)\right)$. Adding in the convex hull of all sets $\eta(\sigma)$ satisfying $\sigma \in \Delta\left(\left[\left(x_{1}, y_{1}\right),\left(x_{2}, y_{2}\right)\right)\right)$ and $\left(x_{1}, y_{1}\right) \in \sigma$ "fills in" the convex hull of the facet $F$ as indicated in figure 3. In the picture, the convex hull of $F \backslash\left\{x_{1}\right\}$ is represented by the line segment connecting $y_{1}$ and $\dot{y}_{1}$, the convex hull of $F \backslash\left\{y_{1}\right\}$ is represented by the line segment connecting $x_{1}$ and $\dot{y}_{1}$, and the facet $F$ is represented by the convex hull of $\left\{x_{1}, y_{1}, \dot{y}_{1}\right\}$. Omitted are from the picture all other elements of $F$, which belong to $F \backslash\left\{x_{1}\right\}$ and $F \backslash\left\{y_{1}\right\}$ as well. Including them would make the picture many dimensional, but would only involve "coning" over each missing vertex.

Using Lemma A.1 we may conclude the proof of the missing special case of Theorem 1.10 as follows. Assume $x_{1}<y_{1} \leq x_{2} \prec y_{2}$ and consider the triangulation of $\triangle\left(\left\{x_{1}\right\} \cup\right.$ $\left.\left[y_{1}, x_{2}\right)\right)$ using the map $\eta:\left(\left(x_{1}, y_{1}\right),\left(x_{2}, y_{2}\right)\right) \rightarrow E$ described in the Lemma. Note that the abstract simplicial complex $\triangle\left(\left\{x_{1}\right\} \cup\left[y_{1}, x_{2}\right)\right)$ is a cone over $x_{1}$ and over $y_{1}$ and that the triangulating complex $\triangle\left(\left[\left(x_{1}, y_{1}\right),\left(x_{2}, y_{2}\right)\right)\right)$ is a cone over $\left(x_{1}, y_{1}\right)$. The map $\eta$ sends $\left(x_{1}, y_{1}\right)$ into the midpoint of the line segment connecting $x_{1}$ and $y_{1}$. Let us restrict now $\eta$ to $\triangle\left(\left(\left(x_{1}, y_{1}\right),\left(x_{2}, y_{2}\right)\right)\right)$. The union of the convex hulls of the sets $\eta(\sigma)$ where $\sigma \in$ $\triangle\left(\left(\left(x_{1}, y_{1}\right),\left(x_{2}, y_{2}\right)\right)\right)$ is the same as the union of the convex hulls of those $\tau \in \triangle\left(\left\{x_{1}\right\} \cup\right.$ $\left.\left[y_{1}, x_{2}\right)\right)$ which do not contain $\eta\left(x_{1}, y_{1}\right)$. It is easy to see that these sets $\tau$ are precisely those elements of $\Delta\left(\left\{x_{1}\right\} \cup\left[y_{1}, x_{2}\right)\right)$ which contain at most one of $x_{1}$ and $y_{1}$, but not both. The family of sets $\left\{\tau \in \triangle\left(\left\{x_{1}\right\} \cup\left[y_{1}, x_{2}\right)\right):\left\{x_{1}, y_{1}\right\} \nsubseteq \tau\right\}$ is isomorphic to the suspension of 


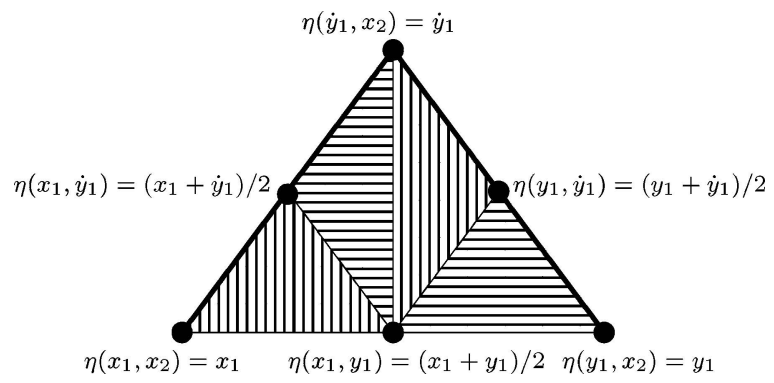

Figure 3. The effect of adding the faces $\eta(\sigma)$ containing $\eta\left(x_{1}, y_{1}\right)$.

$\triangle\left(\left(y_{1}, x_{2}\right)\right)$. (The "suspending" vertices are $x_{1}$ and $y_{1}$.)

We conclude the Appendix with a conjecture generalizing [6, Theorem 5.2].

Conjecture A.2 If the order complex of every open interval in a poset $Q$ is shellable, then the same holds for the order complex of every open interval in $\check{T}(Q)$.

As a consequence of Theorem 1.10 we get a valid statement if we replace "shellable" with "Cohen-Macaulay", since the Cohen-Macaulay property depends only on the geometric realization of the simplicial complex (see [14, Proposition 4.3]). A possible way to attempt proving Conjecture A.2 is trying to generalize the proof of [6, Theorem 5.2] using Lemma 2.3 .

\section{Acknowledgments}

I wish to thank to Professor Ralf Holtkamp for his expert advice on noncommutative formal power series, and to two anonymous referees for improving both the form and the substance of this paper.

\section{Notes}

1. This notation is consistent with the one introduced in [6]. Alas on figure 2 the cover relations labeled with $R$ are vertical and the cover relations labeled with $L$ represent a move northeast. We wish to stress that the letters are meant to indicate the coordinate to be removed and not the direction in a picture.

2. For proof Hoffman cites his earlier work [8] where the treatment of this classical question is embedded into a broader framework. The actual function $1 /(\cos (t)-u \sin (t))$ appears as part of [8, Example 2], where Hoffman mentions the historic source [12]. This reference is older than the papers by Haigh [5] and by Knuth and Buckholtz [11], cited by “The Online Encyclopedia of Integer Sequences” as reference to sequence A008294, which consists of the table of coefficients for the polynomials $Q_{n}(x)$.

\section{References}

1. M. Aigner, "Combinatorial theory," Grundlehren der Mathematischen Wissenschaften, Vol. 234, SpringerVerlag, New York, 1979. 
2. James W. Brewer, "Power series over commutative rings," Lecture Notes in Pure and Applied Mathematics, Vol. 64, Marcel Dekker, New York, 1981.

3. R. Ehrenborg and M. Readdy, "Sheffer posets and r-signed permutations," Ann. Sci. Math. Québec 19 (1995) 173-196.

4. L. Gerritzen and R. Holtkamp, “On Gröbner bases of noncommutative power series,” Indag. Math. (N.S.) 4 (1998) 503-519.

5. G. Haigh, “A natural approach to Pick's theorem,” Math. Gaz. 64 (1980) 173-180.

6. G. Hetyei, "Tchebyshev posets," Discrete \& Comput. Geom. 32 (2004) 493-520.

7. G. Hetyei, "Orthogonal polynomials represented by CW-spheres," Electron. J. Combin. 11(2) (2004) \#R4, 28.

8. M.E. Hoffman, "Derivative polynomials for tangent and secant," Amer. Math. Monthly 102 (1995) 23-30.

9. M.E. Hoffman, "Derivative polynomials, euler polynomials, and associated integer sequences," Electron. J. Comb. 6 (1999) \#R21.

10. R. Holtkamp, personal communication.

11. D.E. Knuth and T.J. Buckholtz, "Computation of tangent, Euler and Bernoulli numbers," Math. Comp. 21 (1967) 663-688.

12. C. Krichnamachary and Rao M. Bhimasena, "On a table for calculating Eulerian numbers based on a new method," Proc. London Math. Soc. 22(2) (1923) 73-80.

13. V. Reiner, "Upper binomial posets and signed permutation statistics," Europ. J. Combin. 14 (1993) 581-588

14. R.P. Stanley, Combinatorics and Commutative Algebra, 2nd ed., Birkhäuser Boston, 1996.

15. R.P. Stanley, Enumerative Combinatorics, Vol. I, Cambridge University Press, Cambridge, 1997.

16. R.P. Stanley, Enumerative Combinatorics, Vol. 2, Cambridge University Press, Cambridge, 1999.

17. R.P. Stanley, "A survey of Eulerian posets," in: Polytopes: Abstract, Convex, and Computational, T. Bisztriczky, P. McMullen, R. Schneider and A.I. Weiss (eds.), NATO ASI Series C, Vol. 440, Kluwer Academic Publishers, 1994, pp. 301-333. 